\title{
Perron's method for viscosity solutions of semilinear path dependent PDEs
}

\author{
Zhenjie REN*
}

August 14, 2018

\begin{abstract}
This paper proves the existence of viscosity solutions of path dependent semilinear PDEs via Perron's method, i.e. via showing that the supremum of viscosity subsolutions is a viscosity solution. We use the notion of viscosity solutions introduced in 9] which considers as test functions all those smooth processes which are tangent in mean. We also provide a comparison result for semicontinuous viscosity solutions, by using a regularization technique. As an interesting byproduct, we give a new short proof for the optimal stopping problem with semicontinuous obstacles.
\end{abstract}

\section{Introduction}

The recently developed theory of viscosity solutions for path dependent PDEs extends the classical notion of viscosity solution of PDEs introduced by Crandall and Lions [ 6 (for an overview we refer to [7] and [1]). Nonlinear path dependent PDEs appear in various applications, such as the stochastic control of non-Markovian systems [10] and the corresponding stochastic differential games [15. They are also intimately related to the backward stochastic differential equations introduced by Pardoux and Peng [13, and their extension to the second order in [4, 19. Loosely speaking, solutions of backward SDEs can be viewed as Sobolev solutions of path-dependent PDEs, and our goal is to develop the alternative notion of viscosity solutions which is well-known to provide a suitable wellposedness and stability theory in the Markovian case $u(t, \omega)=u\left(t, \omega_{t}\right)$.

In the recent work of Ren, Touzi and Zhang [18, the authors focus on the semilinear path dependent PDEs and prove the comparison result for continuous viscosity solutions, in the spirit of the work of Caffarelli and Cabré $[3$ in the context of PDEs. In [9, 18, it is also proved that the solutions of corresponding backward SDEs are viscosity solutions, instead, we are interested in proving the existence of viscosity solutions to semilinear path dependent PDEs by PDE-type arguments, that is, by Perron's method. It is worth noting that in the fully nonlinear case, one

\footnotetext{
${ }^{*}$ CMAP, Ecole Polytechnique Paris, ren@cmap.polytechnique.fr. Research supported by grants from Région Ilede-France
} 
may no longer depend on backward SDEs for finding viscosity solutions for path dependent PDEs, and thus the Perron method will be necessary. Although our result cannot be applied to the fully nonlinear case directly, many arguments in this paper could be useful. Also, the Perron method is not only useful in proving the existence of viscosity solutions, but also has applications in various contexts, for example, the wellposedness of envelope viscosity solution (see [1]), the uniqueness of martingale problems [5], etc. In the proof of Perron's method, we follow the same idea as the classical literature on viscosity solutions of PDEs, but the arguments turn out to be different and nontrivial.

It is well understood in PDE literature that the comparison result for continuous viscosity solutions is not sufficient for the existence of solutions. In Perron's method, we need a comparison result for semicontinuous viscosity solutions. However, the argument in [18 cannot be adapted into our context, because it is not clear whether upper semicontinuous submartingales are almost everywhere punctually differentiable (a crucial intermediate result in [18). In this paper, we apply a regularization on semicontinuous viscosity solutions so as to mollify them to be continuous. Let $u$ be a viscosity subsolution, and $u^{n}$ be its regularized version. A reasonable regularization should satisfy:

$$
u^{n} \text { is continuous; } \quad u^{n} \rightarrow u \text {, as } n \rightarrow \infty ; \quad u^{n} \text { is still a viscosity subsolution. }
$$

The regularization we propose involves a backward distance for paths, is new in literature, satisfies all the above conditions and helps to prove the comparison result. It is worth mentioning that a regularization is probably inevitable in the study of the comparison result for fully nonlinear path dependent PDEs. The regularization we find in this paper might shed light on the future research.

As in the previous work on the viscosity solutions of path dependent PDEs, the optimal stopping result plays a crucial role to overcome the non-local-compactness of the path space. Since we treat semicontinuous viscosity solutions in this paper, we need the corresponding result of optimal stopping under nonlinear expectation for semicontinuous obstacles. In the existing literature, Kobylanski and Quenez [12] contains the desired result but only in the case of linear expectation. Peng and Xu studied in 14 reflected backward SDEs with $L^{2}$ obstacles, and they proved a crucial intermediate result which can lead to the optimal stopping result. However, since their main interest is reflected backward SDEs, there is no direct theorem that we may apply. In this paper, we give a new simple proof for the optimal stopping problem, by using the minimum condition of the Skorokhod decomposition.

The rest of the paper is organized as follows. Section 2 introduces the often used notations. Section 3 recalls the definition of viscosity solutions to path dependent PDEs. Section 4 presents the main results of the paper: the comparison result of semicontinuous viscosity solutions and the result of Perron's method. Then Section 5 contains the proof of Perron's method, and Section 6 is devoted to the comparison result. Section 7 reports the proof of the optimal stopping result. Finally, Section 8 is the appendix in which we complete some proofs. 


\section{Preliminary}

Throughout this paper let $T>0$ be a given finite maturity, $\Omega:=\left\{\omega \in C\left([0, T] ; \mathbb{R}^{d}\right): \omega_{0}=0\right\}$ be the set of continuous paths starting from the origin, and $\Theta:=[0, T] \times \Omega$. We denote $B$ as the canonical process on $\Omega, \mathbb{F}=\left\{\mathcal{F}_{t}, 0 \leq t \leq T\right\}$ as the canonical filtration, $\mathcal{T}$ as the set of all $\mathbb{F}$-stopping times taking values in $[0, T]$. Further let $\mathcal{T}^{+}$denote the subset of $\tau \in \mathcal{T}$ taking values in $(0, T]$, and for $\mathrm{H} \in \mathcal{T}$, let $\mathcal{T}_{\mathrm{H}}$ and $\mathcal{T}_{\mathrm{H}}^{+}$be the subset of $\tau \in \mathcal{T}$ taking values in $[0, \mathrm{H}]$ and in $(0, \mathrm{H}]$, respectively. We also denote $\mathbb{P}_{0}$ as the Wiener measure on $\Omega$, and define the augmented filtration by $\mathbb{F}^{*}:=\left\{\mathcal{F}_{t} \vee \mathcal{N} ; 0 \leq t \leq T\right\}$, where $N$ is the collection of all $\mathbb{P}_{0}$-null sets.

Following Dupire [8], we introduce the following pseudo-distance on $\Theta$ :

$$
\|\omega\|:=\sup _{0 \leq s \leq T}\left|\omega_{s}\right|, \quad d\left(\theta, \theta^{\prime}\right):=\left|t-t^{\prime}\right|+\left\|\omega_{t \wedge}-\omega_{t^{\prime} \wedge}^{\prime}\right\| \quad \text { for all } \quad \theta=(t, \omega), \theta^{\prime}=\left(t^{\prime}, \omega^{\prime}\right) \in \Theta .
$$

We say a process valued in some metric space $E$ is in $C^{0}(\Theta, E)$ whenever it is continuous with respect to $d$. Similarly, $\mathbb{L}^{0}(\mathcal{F}, E)$ and $\mathbb{L}^{0}(\mathbb{F}, E)$ denote the set of $\mathcal{F}$-measurable random variables and $\mathbb{F}$-progressively measurable processes, respectively. We remark that $C^{0}(\Theta, E) \subset \mathbb{L}^{0}(\mathbb{F}, E)$, and when $E=\mathbb{R}$, we shall omit it in these notations.

In this paper, we also use another (backward) pseudo-distance on $\Theta$ :

$$
\overleftarrow{d}\left(\theta, \theta^{\prime}\right):=\left|t-t^{\prime}\right|+\sup _{s \geq 0}\left|\omega_{(t-s) \vee 0}-\omega_{\left(t^{\prime}-s\right) \vee 0}^{\prime}\right|
$$

The following lemma explains the relation between $d(\cdot, \cdot)$ and $\overleftarrow{d}(\cdot, \cdot)$

Lemma 2.1 For all $\theta, \theta^{\prime} \in \Theta$, we have

$$
\left|d\left(\theta, \theta^{\prime}\right)-\overleftarrow{d}\left(\theta, \theta^{\prime}\right)\right| \leq \bar{\rho}\left(\theta,\left|t-t^{\prime}\right|\right), \quad \text { where } \quad \bar{\rho}(\theta, \delta):=\sup _{\left|s-s^{\prime}\right| \leq \delta}\left|\omega_{t \wedge s}-\omega_{t \wedge s^{\prime}}\right|
$$

In particular, a function $f: \Theta \rightarrow \mathbb{R}$ is continuous in $d(\cdot, \cdot)$ if and only if $f$ is continuous in $\overleftarrow{d}(\cdot, \cdot)$

Proof Define $\omega_{s}=0$ for $s<0$. The first claim follows from the simple observation:

$$
|| \omega_{t \wedge s}-\omega_{t^{\prime} \wedge s}^{\prime}|-| \omega_{t \wedge\left(t-t^{\prime}+s\right)}-\omega_{t^{\prime} \wedge s}^{\prime}|| \leq\left|\omega_{t \wedge s}-\omega_{t \wedge\left(t-t^{\prime}+s\right)}\right| \leq \bar{\rho}\left(\theta,\left|t-t^{\prime}\right|\right) .
$$

The second claim is a trivial corollary.

For any $A \in \mathcal{F}_{T}, \xi \in \mathbb{L}^{0}\left(\mathcal{F}_{T}, E\right), X \in \mathbb{L}^{0}(\mathbb{F}, E)$, and $(t, \omega) \in \Theta$, define:

$$
\begin{gathered}
A^{t, \omega}:=\left\{\omega^{\prime} \in \Omega: \omega \otimes_{t} \omega^{\prime} \in A\right\}, \quad \xi^{t, \omega}\left(\omega^{\prime}\right):=\xi\left(\omega \otimes_{t} \omega^{\prime}\right), \quad X_{s}^{t, \omega}\left(\omega^{\prime}\right):=X\left(t+s, \omega \otimes_{t} \omega^{\prime}\right) \\
\text { for all } \omega^{\prime} \in \Omega, \text { where }\left(\omega \otimes_{t} \omega^{\prime}\right)_{s}:=\omega_{s} \mathbf{1}_{[0, t]}(s)+\left(\omega_{t}+\omega_{s-t}^{\prime}\right) \mathbf{1}_{(t, T]}(s), \quad 0 \leq s \leq T .
\end{gathered}
$$

Following the standard arguments of monotone class, we have the following simple results.

Lemma 2.2 Let $0 \leq t \leq s \leq T$ and $\omega \in \Omega$. Then $A^{t, \omega} \in \mathcal{F}_{s-t}$ for all $A \in \mathcal{F}_{s}, \xi^{t, \omega} \in \mathbb{L}^{0}\left(\mathcal{F}_{s-t}, E\right)$ for all $\xi \in \mathbb{L}^{0}\left(\mathcal{F}_{s}, E\right), X^{t, \omega} \in \mathbb{L}^{0}(\mathbb{F}, E)$ for all $X \in \mathbb{L}^{0}(\mathbb{F}, E)$, and $\tau^{t, \omega}-t \in \mathcal{T}_{s-t}$ for all $\tau \in \mathcal{T}_{s}$. 
We consider the semilinear path dependent PDE:

$$
-\mathcal{L} u(\theta)-F\left(\cdot, u, \partial_{\omega} u\right)(\theta)=0, \quad \text { where } \mathcal{L} u:=\partial_{t} u+\frac{1}{2} \partial_{\omega \omega}^{2} u .
$$

Introduce a family of probability measure:

$$
\mathcal{P}:=\left\{\mathbb{P}_{\mu}: \frac{d \mathbb{P}_{\mu}}{d \mathbb{P}_{0}}=\exp \left(\int_{0}^{T} \mu_{t} d B_{t}-\frac{1}{2} \int_{0}^{T} \mu_{t}^{2} d t\right), \text { for some } \mu \in \mathbb{L}^{0}\left(\mathbb{F}, \mathbb{R}^{d}\right),\|\mu\| \leq L\right\},
$$

where $L$ is a constant. Then the corresponding nonlinear expectations are defined as:

$$
\overline{\mathcal{E}}_{L}[\cdot]:=\sup _{\mathbb{P} \in \mathcal{P}} \mathbb{E}^{\mathbb{P}}[\cdot], \quad \underline{\mathcal{E}}_{L}[\cdot]:=\inf _{\mathbb{P} \in \mathcal{P}} \mathbb{E}^{\mathbb{P}}[\cdot] .
$$

\section{Definition of viscosity solution for path dependent PDEs}

As showed in [17, 18, we may define viscosity solutions via semijets. Define the following space of measurable processes:

$$
\mathbf{L}^{1}\left(\mathcal{P}_{L}\right):=\left\{X \in \mathbb{L}^{0}(\mathbb{F}): \overline{\mathcal{E}}_{L}\left[\sup _{s \leq T-t}\left|X_{s}^{\theta}\right|\right]<\infty \text { for all } \theta=(t, \omega) \in \Theta\right\} .
$$

Definition 3.1 (Semijets) For $u \in \mathbf{L}^{1}\left(\mathcal{P}_{L}\right)$, the subjet and superjet of $u$ at $\theta$ are defined as:

$$
\begin{aligned}
& \underline{\mathcal{J}}_{L} u(\theta):=\left\{(\alpha, \beta) \in \mathbb{R} \times \mathbb{R}^{d}: u(\theta)=\max _{\tau \in \mathcal{T}_{\mathrm{H}}} \overline{\mathcal{E}}_{L}\left[u_{\tau}^{\theta}-\alpha \tau-\beta B_{\tau}\right], \text { for some } \mathrm{H} \in \mathcal{T}^{+}\right\} ; \\
& \overline{\mathcal{J}}_{L} u(\theta):=\left\{(\alpha, \beta) \in \mathbb{R} \times \mathbb{R}^{d}: u(\theta)=\min _{\tau \in \mathcal{T}_{\mathrm{H}}} \underline{\mathcal{E}}_{L}\left[u_{\tau}^{\theta}-\alpha \tau-\beta B_{\tau}\right], \text { for some } \mathrm{H} \in \mathcal{T}^{+}\right\} .
\end{aligned}
$$

Definition 3.2 (Viscosity solution) Let $u \in \mathbf{L}^{1}\left(\mathcal{P}_{L}\right)$. Then,

(i) $u$ is a viscosity subsolution (resp. supersolution) of the path dependent PDE (2.2), if for all $\theta \in \Theta$, and $(\alpha, \beta) \in \underline{\mathcal{J}}_{L} u(\theta)$ (resp. $\overline{\mathcal{J}}_{L} u(\theta)$ ), it holds that

$$
-\alpha-F(\theta, u(\theta), \beta) \leq(\text { resp. } \geq) 0 .
$$

(ii) $u$ is a viscosity solution of the path dependent PDE (2.2), if $u$ is both a viscosity subsolution and a viscosity supersolution.

Remark 3.3 The definition of viscosity solutions depends on the constant $L$. In [18, the authors give the name as $\mathcal{P}_{L}$-viscosity sub-/super-solutions. For the simplification of notations, we simply call them viscosity sub-/super-solutions in this paper.

\section{Main results}

\subsection{Comparison result for semicontinuous viscosity solutions}

In [18, a comparison result is proved for continuous viscosity solutions. In this paper, we provide an extension to semicontinuous viscosity solutions, which plays an important role in the Perron approach. 
Assumption 4.1 The generator function $F(\theta, y, z)$ satisfies the following assumptions.

(i) $F$ is uniformly Lipschitz continuous in $(y, z)$, i.e. there exists a constant $L$ such that

$$
\left|F(\cdot, y, z)-F\left(\cdot, y^{\prime}, z^{\prime}\right)\right| \leq L\left|y-y^{\prime}\right|+L\left|z-z^{\prime}\right|
$$

(ii) There exists $F^{0} \in C^{0}(\Theta)$ such that $|F(\cdot, 0,0)| \leq F^{0}$.

(iii) There exists a function $\rho^{F}:(\theta, x, y) \in \Theta \times \mathbb{R} \times \mathbb{R} \longrightarrow \mathbb{R}$ such that $\rho^{F}$ is continuous in $(\theta, x, y)$ and non-decreasing in $\gamma, \rho^{F}(\theta, 0, y)=0$ for all $(\theta, y) \in \Theta \times \mathbb{R}$, and

$$
\left|F(\theta, y, \cdot)-F\left(\theta^{\prime}, y, \cdot\right)\right| \leq \rho^{F}\left(\theta, d\left(\theta, \theta^{\prime}\right), y\right), \quad \text { for all } \theta, \theta^{\prime} \in \Theta
$$

Our comparison result is based on the following consequence of Theorem $4.1 \mathrm{in}$ Ren, Touzi and Zhang [18.

Theorem 4.2 Let Assumption 4.1 (i) and (ii) hold true, and $u, v \in C^{0}(\Theta)$ be bounded viscosity subsolution and supersolution of path dependent PDE (2.2), respectively. If $u_{T} \leq v_{T}$, then $u \leq v$ on $\Theta$.

Remark 4.3 The comparison result in 18 is established fo continuous viscosity subsolutions and supersolutions which do not need to be bounded, but satisfy some integrability condition. In Assumption 4.1, the first two are the same as the assumption in [18], while (iii) is the extra assumption for the comparison result of semicontinuous viscosity solutions.

Definition 4.4 A function $u: \Theta \rightarrow \mathbb{R}$ belongs to $\mathrm{USC}_{b}$ (resp. $\mathrm{LSC}_{b}$ ), if $u$ is bounded and satisfies

$$
u(\theta) \geq \varlimsup_{d\left(\theta, \theta^{\prime}\right) \rightarrow 0} u\left(\theta^{\prime}\right) \quad\left(\text { resp. } \leq \varliminf_{d\left(\theta_{\left.\theta, \theta^{\prime}\right) \rightarrow 0}\right.} u\left(\theta^{\prime}\right)\right) .
$$

We will prove in Section 6 that

Theorem 4.5 Let Assumption 4.1 hold true, and $u \in \mathrm{USC}_{b}(\Theta), v \in \mathrm{LSC}_{b}(\Theta)$ be viscosity subsolution and supersolution of path dependent PDE (2.2), respectively. If $u_{T} \leq v_{T}$, then $u \leq v$ on $\Theta$.

Remark 4.6 The argument of proving the comparison result for continuous viscosity solutions in [18 cannot be adapted directly to our context, because it is not clear whether a USC submartingale is almost everywhere punctually differentiable (see the definition in [18). Our strategy is to apply a regularization so as to introduce continuous approximations which are still viscosity sub-/supersolutions, and then we apply the comparison result for continuous viscosity solutions. Let $u$ be a viscosity subsolution, and $u^{n}$ be its regularized version. A reasonable regularization should satisfy:

$$
u^{n} \text { is continuous; } \quad u^{n} \rightarrow u \text {, as } n \rightarrow \infty ; \quad u^{n} \text { is still a viscosity subsolution. }
$$

The regularization introduced in Section 6.1 satisfies all above conditions, and helps to prove the comparison result. 


\subsection{Existence via Perron's method}

Due to Proposition 3.14 in [10], we may equivalently study the existence of viscosity solution for the equation corresponding to the change of variable: $\tilde{u}_{t}:=e^{-L t} u_{t}$. It follows from the Lipschitz property of the nonlinearity $F$ in $y$ that we may assume without loss of generality that $F$ is increasing in $y$.

Assumption 4.7 The generator function $F(\theta, y, z)$ satisfies (i) of Assumptions 4.1 and:

(i) $F$ is continuous in $\theta$.

(ii) $F$ is non-decreasing in $y$.

For a function $w$ on $\Theta$, we define its USC and LSC envelops:

$$
w^{*}(\theta):=\varlimsup_{d\left(\theta, \theta^{\prime}\right) \rightarrow 0} w\left(\theta^{\prime}\right) \quad \text { and } \quad w_{*}(\theta):=\varliminf_{d\left(\theta, \theta^{\prime}\right) \rightarrow 0} w\left(\theta^{\prime}\right) .
$$

We will prove in Section 5 that:

Theorem 4.8 Let Assumption 4.7 and the comparison result of Theorem 4.5 hold true. Assume further that there is a viscosity subsolution $\underline{u} \in \operatorname{USC}_{b}(\Theta)$ and a supersolution $\bar{v} \in \operatorname{LSC}_{b}(\Theta)$ of Equation (2.2) which satisfy the boundary condition $\left(\underline{u}_{*}\right)_{T}=\bar{v}_{T}^{*}=\xi$. Denote

$$
\mathcal{D}:=\left\{\phi: \phi \in \mathrm{USC}_{b}(\Theta) \text { is a viscosity suboslution of Equation (2.2) and } \underline{u} \leq \phi \leq \bar{v}\right\} .
$$

Then $u(\theta):=\sup \{\phi(\theta): \phi \in \mathcal{D}\}$ is a continuous viscosity solution of Equation (2.2), and satisfies the boundary condition $u_{T}=\xi$.

\section{Perron's method}

We will prove in the following subsections the two propositions:

Proposition $5.1 u^{*} \in \mathrm{USC}_{b}(\Theta)$ is a viscosity subsolution of Equation (2.2).

Proposition $5.2 u_{*} \in \operatorname{LSC}_{b}(\Theta)$ is a viscosity supersolution of Equation (2.2).

Then the comparison result allows to complete the proof.

Proof of Theorem 4.8 Since $u \geq \underline{u}$, we have $u_{*} \geq \underline{u}_{*}$, in particular, $\left(u_{*}\right)_{T} \geq \xi$. On the other hand, since $u \leq \bar{v}$, we have $u^{*} \leq \bar{v}^{*}$, in particular, $u_{T}^{*} \leq \xi$. Therefore, $u_{T}^{*} \leq\left(u_{*}\right)_{T}$, and it follows from the comparison result that $u^{*} \leq u_{*}$. We conclude that $u^{*}=u=u_{*}$, and thus $u$ is a bounded continuous viscosity solution of Equation (2.2). 


\subsection{Some useful lemmas}

As in [9, 17, 18, the optimal stopping result is crucial in the current theory of viscosity solution to path dependent PDE. As we are going to treat semicontinuous viscosity solutions, we need an optimal stopping result for semicontinuous obstacles.

Definition 5.3 (i) $A$ random variable $X$ is $\overline{\mathcal{E}}_{L}$-uniformly integrable if

$$
\lim _{A \rightarrow \infty} \overline{\mathcal{E}}_{L}[|X| ;|X| \geq A] \rightarrow 0 .
$$

(ii) A family of random variables $\left\{X_{\alpha}\right\}$ is $\overline{\mathcal{E}}_{L}$-uniformly integrable if

$$
\lim _{A \rightarrow \infty} \sup _{\alpha} \overline{\mathcal{E}}_{L}\left[\left|X_{\alpha}\right| ;\left|X_{\alpha}\right| \geq A\right] \rightarrow 0 .
$$

One may easily prove the following two lemmas.

Lemma 5.4 (Dominated convergence) Let $X_{n}$ be a sequence of r.v.'s such that $\left\{X_{n}\right\}_{n}$ is $\overline{\mathcal{E}}_{L^{-}}$ uniformly integrable, and $X_{n} \rightarrow 0, \mathbb{P}_{0}$-a.s. Then we have $\lim _{n \rightarrow \infty} \overline{\mathcal{E}}_{L}\left[\left|X_{n}\right|\right]=0$.

Lemma 5.5 (Fatou's lemma) Let $X_{n}$ be a sequence of bounded r.v.'s. Then we have

$$
\varlimsup_{n \rightarrow \infty} \overline{\mathcal{E}}_{L}\left[X_{n}\right] \leq \overline{\mathcal{E}}_{L}\left[\varlimsup_{n \rightarrow \infty} X_{n}\right] .
$$

Denote by $\mathcal{T}_{*}$ the set of all $\mathbb{F}^{*}$-stopping times

Theorem 5.6 (Optimal stopping for semicontinuous obstacle) Let $X$ be an $\mathbb{F}^{*}$-progressively measurable process such that

(i) $X$ is upper semicontinuous (u.s.c.) in $t, \mathbb{P}_{0}$-a.s.;

(ii) $\sup _{t} X_{t}^{+}$is $\overline{\mathcal{E}}_{L}$-uniformly integrable;

(iii) $X_{t}^{-}$is $\overline{\mathcal{E}}_{L}$-uniformly integrable for all $t \in[0, T]$.

Define

$$
Y(\theta)=\sup _{\tau \in \mathcal{T}_{*}} \overline{\mathcal{E}}_{L}\left[X_{\tau}^{\theta}\right]
$$

Then there exits a stopping time $\tau^{*} \in \mathcal{T}_{*}$ such that $Y_{0}=\overline{\mathcal{E}}_{L}\left[X_{\tau^{*}}\right]$ and $X_{\tau^{*}}=Y_{\tau^{*}}, \mathbb{P}_{0}$-a.s.

This theorem will be proved in Section 7. Based on Theorem 5.6, we may prove the following lemma similar to Lemma 4.9 in [18], but concerning pathwise u.s.c. functions.

Lemma 5.7 Let $u_{\wedge_{\wedge \mathrm{H}}}$ satisfy the assumptions in Theorem [5.6 and assume that $u_{0}>\overline{\mathcal{E}}_{L}\left[u_{\mathrm{H}}\right]$ for some $\mathrm{H} \in \mathcal{T}^{+}$. Then there exists $\omega^{*} \in \Omega$ and $t^{*}<\mathrm{H}\left(\omega^{*}\right)$ such that $(0,0) \in \underline{\mathcal{I}}_{L} u\left(\theta^{*}\right)$.

Proof Define the optimal stopping problem $Y$ by (5.1) with $X:=u_{\wedge \wedge \mathrm{H}}$. Let $\tau^{*} \in \mathcal{T}_{*}$ be the optimal stopping rule. By Theorem 5.6 we have

$$
\overline{\mathcal{E}}_{L}\left[u_{\tau^{*}}\right]=Y_{0} \geq u_{0}>\overline{\mathcal{E}}_{L}\left[u_{\mathrm{H}}\right] \text { and } \mathbb{P}_{0}\left[u_{\tau^{*}}=Y_{\tau^{*}}\right]=1,
$$

and it follows that $\mathbb{P}_{0}\left[u_{\tau^{*}}=Y_{\tau^{*}}, \tau^{*}<\mathrm{H}\right]>0$. Then there exists $\omega^{*} \in \Omega$ such that $t^{*}:=\tau^{*}\left(\omega^{*}\right)<$ $\mathrm{H}\left(\omega^{*}\right)$ and $u_{t^{*}}\left(\omega^{*}\right)=Y_{t^{*}}\left(\omega^{*}\right)$. Therefore, $\left(t^{*}, \omega^{*}\right)$ is the desired point.

The next result about semijet is useful for proving stability results. 
Lemma 5.8 Let $u \in \mathbb{L}^{0}(\mathbb{F})$ be bounded, and $u^{n} \in \mathbb{L}^{0}(\mathbb{F})$ be bounded and pathwise u.s.c. $\mathbb{P}_{0}$-a.s. Fix $\theta \in \Theta$, and suppose that

(i) there exists a sequence $\left\{\theta^{n}\right\} \subset \Theta$ such that

$$
d\left(\theta^{n}, \theta\right) \rightarrow 0 \quad \text { and } \quad u(\theta)=\lim _{n \rightarrow \infty} u^{n}\left(\theta^{n}\right)
$$

(ii) for any $\bar{\theta} \in \Theta$ and any sequence $\left\{\bar{\theta}^{n}\right\} \subset \Theta$ such that $d\left(\bar{\theta}^{n}, \bar{\theta}\right) \rightarrow 0$, it holds

$$
u(\bar{\theta}) \geq \varlimsup_{n \rightarrow \infty} u^{n}\left(\bar{\theta}^{n}\right) .
$$

Then, for any $(\alpha, \beta) \in \underline{\mathcal{J}}_{L} u(\theta), n \in \mathbb{N}$ and $\varepsilon>0$, there exits $N_{n} \geq n$ and $\hat{\theta}^{n}$ such that

$$
d\left(\theta^{N_{n}}, \hat{\theta}^{n}\right) \leq \frac{1}{n} \quad \text { and } \quad(\alpha+\varepsilon, \beta) \in \operatorname{cl}\left(\underline{\mathcal{J}}_{L} u^{N_{n}}\left(\hat{\theta}^{n}\right)\right) .
$$

Proof Since $(\alpha, \beta) \in \underline{\mathcal{J}}_{L} u(\theta)$, there exists $\mathrm{H} \in \mathcal{T}^{+}$such that $u(\theta)=\max _{\tau \in \mathcal{T}_{\mathrm{H}}} \overline{\mathcal{E}}_{L}\left[u_{\tau}^{\theta}-\alpha \tau-\beta B_{\tau}\right]$. Denote $\mathrm{H}^{n}\left(\omega^{\prime}\right):=\mathrm{H}\left(\omega^{\prime}\right) \wedge \inf \left\{t^{\prime}:\left\|\omega_{t^{\prime}}^{\prime}\right\|>\frac{1}{n}\right\}$. Then for any $\varepsilon>0$, it holds

$$
u(\theta)>\overline{\mathcal{E}}_{L}\left[u_{\mathrm{H}^{n}}^{\theta}-(\alpha+\varepsilon) \mathrm{H}^{n}-\beta B_{\mathrm{H}^{n}}\right] .
$$

Further, by (i) and (ii), we obtain

$\lim _{m \rightarrow \infty} u^{m}\left(\theta^{m}\right)>\overline{\mathcal{E}}_{L}\left[\varlimsup_{m \rightarrow \infty}\left(u^{m}\right)_{\mathrm{H}^{n}}^{\theta^{m}}-(\alpha+\varepsilon) \mathrm{H}^{n}-\beta B_{\mathrm{H}^{n}}\right] \geq \varlimsup_{m \rightarrow \infty} \overline{\mathcal{E}}_{L}\left[\left(u^{m}\right)_{\mathrm{H}^{n}}^{\theta^{m}}-(\alpha+\varepsilon) \mathrm{H}^{n}-\beta B_{\mathrm{H}^{n}}\right]$.

Therefore, for each $n$, there exists $N_{n} \geq n$ such that

$$
u^{N_{n}}\left(\theta^{N_{n}}\right)>\overline{\mathcal{E}}_{L}\left[\left(u^{N_{n}}\right)_{\mathrm{H}^{n}}^{\hat{N}_{n}}-(\alpha+\varepsilon) \mathrm{H}^{n}-\beta B_{\mathrm{H}^{n}}\right] .
$$

Then, by Lemma 5.7, we may find $\hat{\theta}^{n}$ such that

$$
d\left(\hat{\theta}^{n}, \theta^{N_{n}}\right) \leq \frac{1}{n} \quad \text { and } \quad(\alpha+\varepsilon, \beta) \in \underline{\mathcal{J}}_{L} u^{N_{n}}\left(\hat{\theta}^{n}\right) .
$$

To finish this subsection, we study a special path dependent PDE, and give one of its viscosity solutions by a stochastic representation. Let $u$ be a bounded process and $\mathrm{H} \in \mathcal{H}$, and define a function:

$$
\eta(\theta):=\underline{\mathcal{E}}_{L}\left[\left(u_{\mathrm{H}}\right)^{\theta}-\alpha\left(\mathrm{H}^{\theta}-t\right)-\beta B_{\mathrm{H}^{\theta}-t}\right] .
$$

Proposition 5.9 (i) $\eta$ is a viscosity subsolution of the path dependent PDE:

$$
-\mathcal{L} \eta(\theta)+\alpha+L\left|\beta-\partial_{\omega} \eta(\theta)\right|=0 .
$$

(ii) If $u$ is Lipschitz continuous, then $\eta$ is continuous on $\{\theta: t \leq \mathrm{H}(\omega)\}$.

We will report the proof in the appendix. 


\subsection{Equivalent definitions of viscosity solution}

Denote by $\mathcal{H}$ the collection of all the stopping times of the form of $\mathrm{H}^{s, O}:=\inf \{t \geq 0: \theta \notin$ $[0, s) \times O\} \in \mathcal{T}^{+}$, where $s>0$ and $O \subset \mathbb{R}^{d}$ is a bounded open convex set.

Definition 5.10 For $u \in \mathbf{L}^{1}\left(\mathcal{P}_{L}\right)$, we define for each $\theta \in \Theta$ :

$$
\begin{aligned}
& \underline{\mathcal{J}}_{L}^{\prime} u(\theta):=\left\{(\alpha, \beta) \in \mathbb{R} \times \mathbb{R}^{d}: u(\theta)=\max _{\tau \in \mathcal{T}_{\mathrm{H}}} \overline{\mathcal{E}}_{L}\left[u_{\tau}^{\theta}-\alpha \tau-\beta B_{\tau}\right], \text { for some } \mathrm{H} \in \mathcal{H}\right\} ; \\
& \overline{\mathcal{J}}_{L}^{\prime} u(\theta):=\left\{(\alpha, \beta) \in \mathbb{R} \times \mathbb{R}^{d}: u(\theta)=\min _{\tau \in \mathcal{T}_{\mathrm{H}}} \underline{\mathcal{E}}_{L}\left[u_{\tau}^{\theta}-\alpha \tau-\beta B_{\tau}\right], \text { for some } \mathrm{H} \in \mathcal{H}\right\} .
\end{aligned}
$$

Comparing to Definition 3.1, we replace the stopping time $\mathrm{H} \in \mathcal{T}^{+}$by a hitting time in $\mathcal{H}$.

Proposition 5.11 Suppose that $u \in \mathrm{USC}_{b}(\Theta)$ and that the generator $F:(\theta, y, z) \mapsto \mathbb{R}$ satisfies Assumption 4.7. Then $u$ is a viscosity subsolution of Equation (2.2) if and only if

$$
-\alpha-F(\theta, u(\theta), \beta) \leq 0, \quad \text { for all } \theta \in \Theta,(\alpha, \beta) \in \underline{\mathcal{J}}_{L}^{\prime} u(\theta) .
$$

The similar result holds for supersolutions.

Proof The 'only if' part is trivial by the definitions. We will only prove the 'if' part. Fix a $\theta \in \Theta$, and suppose $(\alpha, \beta) \in \underline{\mathcal{J}}_{L} u(\theta)$, i.e.

$$
u(\theta)=\max _{\tau \in \mathcal{T}_{\mathrm{H}}} \overline{\mathcal{E}}_{L}\left[u_{\tau}^{\theta}-\alpha \tau-B_{\tau}\right] \text { for some } \mathrm{H} \in \mathcal{T}^{+} .
$$

For any $\delta>0$, we may suppose $\mathrm{H}<\hat{\mathrm{H}}_{\delta}:=\inf \left\{t^{\prime}: d\left(\theta^{\prime}, 0\right) \geq \delta\right\}$. Then for any $\varepsilon>0$ it holds

$$
u(\theta)>\overline{\mathcal{E}}_{L}\left[u_{\mathrm{H}}^{\theta}-(\alpha+\varepsilon) \mathrm{H}-\beta B_{\mathrm{H}}\right] .
$$

We next define a sequence of hitting time:

$$
\overline{\mathrm{H}}_{0}^{n}:=0, \quad \overline{\mathrm{H}}_{k+1}^{n}:=\left(\overline{\mathrm{H}}_{k}^{n}+\frac{1}{n}\right) \wedge \inf \left\{t^{\prime} \geq \overline{\mathrm{H}}_{k}^{n}:\left|\omega_{t^{\prime}}^{\prime}-\omega_{\overline{\mathrm{H}}_{k}^{n}}^{\prime}\right| \geq \frac{1}{n}\right\}, \text { for all } k \geq 0,
$$

and define $\mathrm{H}_{n}:=\inf \left\{\overline{\mathrm{H}}_{k}^{n}: \overline{\mathrm{H}}_{k}^{n}>\mathrm{H}\right\}$. Clearly $\mathrm{H}_{n} \downarrow \mathrm{H}$. Since $u \in \mathrm{USC}_{b}(\Theta)$, it follows from Fatou's Lemma (Lemma 5.5) that

$\overline{\mathcal{E}}_{L}\left[u_{\mathrm{H}}^{\theta}-(\alpha+\varepsilon) \mathrm{H}-B_{\mathrm{H}}\right] \geq \overline{\mathcal{E}}_{L}\left[\varlimsup_{n \rightarrow \infty}\left(u_{\mathrm{H}_{n}}^{\theta}-(\alpha+\varepsilon) \mathrm{H}_{n}-B_{\mathrm{H}_{n}}\right)\right] \geq \varlimsup_{n \rightarrow \infty} \overline{\mathcal{E}}_{L}\left[u_{\mathrm{H}_{n}}^{\theta}-(\alpha+\varepsilon) \mathrm{H}_{n}-B_{\mathrm{H}_{n}}\right]$.

So there exists $n$ sufficiently large such that

$$
u(\theta)>\overline{\mathcal{E}}_{L}\left[u_{\mathrm{H}_{n}}^{\theta}-(\alpha+\varepsilon) \mathrm{H}_{n}-B_{\mathrm{H}_{n}}\right] .
$$

By Lemma 5.7, there exists $\theta^{*} \in \Theta$ such that $t^{*}<\mathrm{H}_{n}\left(\omega^{*}\right)$ and

$$
u\left(\theta^{*}\right)=\max _{\tau \in \mathcal{T}_{\mathrm{H}_{n}^{\theta^{*}}}} \overline{\mathcal{E}}_{L}\left[u_{\tau}^{\theta^{*}}-(\alpha+\varepsilon) \tau-B_{\tau}\right] .
$$

Note that if $\overline{\mathrm{H}}_{k}^{n}\left(\theta^{*}\right) \leq t^{*}<\overline{\mathrm{H}}_{k+1}^{n}\left(\theta^{*}\right)$, then $\mathrm{H}_{n}^{\theta^{*}}-t^{*} \geq \mathrm{H}^{*}:=\left(\overline{\mathrm{H}}_{k+1}^{n}\right)^{\theta^{*}}-t^{*} \in \mathcal{H}$. It follows that

$$
u\left(\theta^{*}\right)=\max _{\tau \in \mathcal{T}_{\mathrm{H}^{*}}} \overline{\mathcal{E}}_{L}\left[u_{\tau}^{\theta^{*}}-(\alpha+\varepsilon) \tau-B_{\tau}\right] .
$$

By (5.2), we obtain that

$$
-(\alpha+\varepsilon)-F\left(\theta^{*}, u\left(\theta^{*}\right), \beta\right) \leq 0 .
$$

Finally, by letting $\delta, \varepsilon \rightarrow 0$ and $n \rightarrow \infty$, we obtain: $-\alpha-F(\theta, u(\theta), \beta) \leq 0$. 


\subsection{Subsolution property}

Proof of Proposition 5.1 Fix any $\theta \in \Theta$. By the definition of $u$ and $u^{*}$, there is a sequence of functions $\left\{\phi^{n}\right\} \subset \mathcal{D}$ and a sequence $\left\{\theta^{n}\right\} \subset \Theta$ such that

$$
d\left(\theta^{n}, \theta\right) \rightarrow 0 \quad \text { and } \quad u^{*}(\theta)=\lim _{n \rightarrow \infty} \phi^{n}\left(\theta^{n}\right)
$$

Then by Lemma 5.8, for any $(\alpha, \beta) \in \underline{\mathcal{J}}_{L} u(\theta), n \in \mathbb{N}$ and $\varepsilon>0$, there is $N_{n} \geq n$ and $\hat{\theta}^{n}$ such that

$$
d\left(\theta^{N_{n}}, \hat{\theta}^{n}\right) \leq \frac{1}{n} \quad \text { and } \quad(\alpha+\varepsilon, \beta) \in \operatorname{cl}\left(\underline{\mathcal{J}}_{L} \phi^{N_{n}}\left(\hat{\theta}^{n}\right)\right) .
$$

Further, since $\phi^{n}(\leq u)$ is a viscosity subsolution of Equation (2.2) for each $n$, we deduce from the non-decrease of $F$ in $y$ that

$$
-(\alpha+\varepsilon)-F\left(\hat{\theta}^{n}, u\left(\hat{\theta}^{n}\right), \beta\right) \leq-(\alpha+\varepsilon)-F\left(\hat{\theta}^{n}, \phi^{N_{n}}\left(\hat{\theta}^{n}\right), \beta\right) \leq 0 .
$$

Then since $\varlimsup_{n \rightarrow \infty} u\left(\hat{\theta}^{n}\right) \leq u^{*}(\theta)$, by letting $n \rightarrow \infty$ we obtain that

$$
-(\alpha+\varepsilon)-F\left(\theta, u^{*}(\theta), \beta\right) \leq 0 .
$$

Finally, by letting $\varepsilon \rightarrow 0$, we get the desired result.

Proposition 5.12 It holds that $u=u^{*} \in \mathrm{USC}_{b}(\Theta)$ is a viscosity subsolution of Equation (2.2).

Proof By the previous proposition, we know that $u^{*} \in \mathcal{D}$, and thus $u^{*} \leq u$. On the other hand, by the definition of $u^{*}$, it holds that $u^{*} \geq u$. Therefore, $u=u^{*}$.

\subsection{Supersolution property}

Proof of Proposition 5.2 1. Suppose that $u_{*}$ is not a viscosity supersolution. Then by Proposition 5.11, there is $\theta^{0}=\left(t^{0}, \omega^{0}\right) \in \Theta$ and $(\alpha, \beta) \in \overline{\mathcal{J}}_{L}^{\prime} u_{*}\left(\theta^{0}\right)$, i.e. $u_{*}\left(\theta^{0}\right)=\min _{\tau \in \mathcal{T}_{\text {H }}} \underline{\mathcal{E}}_{L}\left[\left(u_{*}\right)_{\tau}^{\theta^{0}}-\alpha \tau-\right.$ $\left.\beta B_{\tau}\right]$ for some $\mathrm{H} \in \mathcal{H}$, such that

$$
-\alpha-F\left(\theta^{0}, u_{*}\left(\theta^{0}\right), \beta\right)=: \quad-2 \delta<0 .
$$

Since $F(\theta, y, z)$ is non-decreasing in $y$ and $u_{*} \in \operatorname{LSC}_{b}(\Theta)$, it follows from (5.3) that

$$
-\alpha+\delta-F\left(\cdot, u_{*}, \beta\right)<0 \quad \text { on } O_{9 \varepsilon_{0}}:=\left\{\theta: d\left(\theta^{0}, \theta\right)<9 \varepsilon_{0}\right\} \text { for some small } \varepsilon_{0}>0
$$

Without loss of generality, we may assume that $\mathrm{H}$ is in the form of:

$$
\mathrm{H}(\omega)=3 \varepsilon_{1} \wedge \inf \left\{s:\left|\omega_{s}\right| \geq 3 \varepsilon_{1}\right\} \quad \text { for some } \varepsilon_{1}>0 \text { such that } 3 \varepsilon_{1}<3 \varepsilon_{0} \wedge \rho_{\theta^{0}}^{-1}\left(3 \varepsilon_{0}\right),
$$

where $\rho_{\theta^{0}}$ is an invertible modulus of continuity of the path $\omega^{0}$, and $\rho_{\theta^{0}}^{-1}$ is the inverse function. Further, take a small neighborhood $O_{\varepsilon_{2}}$ of $\theta^{0}$, where

$$
\varepsilon_{2}<\varepsilon_{1} \wedge \rho_{\theta^{0}}^{-1}\left(\varepsilon_{1}\right) .
$$


We next introduce two stopping times:

$$
\mathrm{H}_{0}(\omega):=\inf \left\{t \geq 0: \theta \in O_{\varepsilon_{2}}\right\} \quad \text { and } \quad \mathrm{H}_{1}(\omega):=\inf \left\{t \geq \mathrm{H}_{0}(\omega):\left|\omega_{t}-\omega_{t^{0}}^{0}\right| \geq 3 \varepsilon_{1}\right\} \wedge\left(t^{0}+3 \varepsilon_{1}\right) \text {, }
$$

together with the set:

$$
Q:=\left\{\theta \in \Theta: \mathrm{H}_{0}(\omega) \leq t \leq \mathrm{H}_{1}(\omega)\right\}
$$

We claim and will prove in Step 5 that

$$
O_{\varepsilon_{2}} \subset Q \subset O_{9 \varepsilon_{0}}
$$

In particular, we have $\mathrm{H}_{0}\left(\omega^{0}\right)<t^{0}<\mathrm{H}_{1}\left(\omega^{0}\right)$, and thus $\mathrm{H}_{1}^{\theta^{0}}-t^{0}=\mathrm{H}$. Since $(\alpha, \beta) \in \overline{\mathcal{J}}_{L} u_{*}(\theta)$, we have

$$
u_{*}\left(\theta^{0}\right)<\underline{\mathcal{E}}_{L}\left[\left(u_{*}\right)_{\mathrm{H}_{1}^{\theta^{0}}-t^{0}}^{\theta^{0}}-(\alpha-\delta)\left(\mathrm{H}_{1}^{\theta^{0}}-t^{0}\right)-\beta B_{\mathrm{H}_{1}^{\theta^{0}}-t^{0}}\right] .
$$

We next define the inf-convolution of $u_{*}$ :

$$
\underline{u}^{n}(\theta):=\inf _{\theta^{\prime} \in \Theta}\left\{u_{*}\left(\theta^{\prime}\right)+n d\left(\theta^{\prime}, \theta\right)\right\} \quad \text { for all } \theta \in \Theta .
$$

Notice that $\underline{u}^{n}$ is Lipschitz continuous. Since $u_{*} \in \operatorname{LSC}_{b}(\Theta)$, it is easy to show that $\underline{u}^{n} \uparrow u_{*}$. Thus, by (5.5), we deduce that for $n$ sufficiently large

$$
u_{*}\left(\theta^{0}\right)<\underline{\mathcal{E}}_{L}\left[\left(\underline{u}^{n}\right)_{\mathrm{H}_{1}^{\theta^{0}}-t^{0}}^{\theta^{0}}-(\alpha-\delta)\left(\mathrm{H}_{1}^{\theta^{0}}-t^{0}\right)-\beta B_{\mathrm{H}_{1}^{\theta^{0}}-t^{0}}\right] .
$$

By defining $\varphi(\theta):=\underline{\mathcal{E}}_{L}\left[\left(\underline{u}^{n}\right)_{\mathrm{H}_{1}^{\theta}-t}^{\theta}-(\alpha-\delta)\left(\mathrm{H}_{1}^{\theta}-t\right)-\beta B_{\mathrm{H}_{1}^{\theta}-t}\right]$ for all $\theta \in \Theta$, we have

$$
\varphi\left(\theta^{0}\right)>u_{*}\left(\theta^{0}\right)
$$

We finally define

$$
U:=(\varphi \vee u) 1_{Q}+u 1_{Q^{c}}
$$

2. In this step, we show that $\varphi$ is viscosity subsolution of the equation:

$$
-\mathcal{L} w-F\left(\cdot, \varphi \vee u, \partial_{\omega} w\right) \leq 0, \quad \text { on }\left\{\theta: t<\mathrm{H}_{1}(\omega)\right\} .
$$

It follows from Proposition 5.9 that for all $\left(\alpha^{\prime}, \beta^{\prime}\right) \in \underline{\mathcal{J}}_{L} \varphi(\theta)$, it holds that

$$
-\alpha^{\prime}+\alpha-\delta+L\left|\beta-\beta^{\prime}\right| \leq 0 .
$$

Further, by (5.4) we obtain that

$$
-\alpha^{\prime}-F\left(\theta,(\varphi \vee u)(\theta), \beta^{\prime}\right) \leq-\alpha^{\prime}-F\left(\theta, u_{*}(\theta), \beta\right)+L\left|\beta-\beta^{\prime}\right| \leq 0 .
$$

So the desired result follows. 
3. In this step, we prove that $U$ is a viscosity subsolution of Equation (2.2). First, for $\theta \in Q^{o}:=$ $\left\{\theta: \mathrm{H}_{0}(\omega) \leq t<\mathrm{H}_{1}(\omega)\right\}$, it is clear that both $\varphi$ and $u$ are viscosity subsolutions of Equation (5.7). Then take any $\left(\alpha^{\prime}, \beta^{\prime}\right) \in \underline{\mathcal{J}}_{L} U(\theta)$, i.e.

$$
U(\theta)=\max _{\tau \in \mathcal{T}_{\mathrm{H}^{\prime}}} \overline{\mathcal{E}}_{L}\left[U_{\tau}^{\theta}-\alpha^{\prime} \tau-\beta^{\prime} B_{\tau}\right] \quad \text { for some } \quad \mathrm{H}^{\prime} \in \mathcal{T}^{+} .
$$

If $u(\theta) \leq \varphi(\theta)$, then it follows that

$$
\varphi(\theta) \geq \overline{\mathcal{E}}_{L}\left[U_{\tau}^{\theta^{\prime}}-\alpha^{\prime} \tau-\beta^{\prime} B_{\tau}\right] \geq \overline{\mathcal{E}}_{L}\left[\varphi_{\tau}^{\theta^{\prime}}-\alpha^{\prime} \tau-\beta^{\prime} B_{\tau}\right] \text { for all } \tau \in \mathcal{T}_{\mathrm{H}^{\prime}} .
$$

Thus $\left(\alpha^{\prime}, \beta^{\prime}\right) \in \underline{\mathcal{J}}_{L} \varphi(\theta)$. Otherwise, if $u(\theta)>\varphi(\theta)$, we may similarly get $\left(\alpha^{\prime}, \beta^{\prime}\right) \in \underline{\mathcal{J}}_{L} u(\theta)$. In both cases, it follows that

$$
-\alpha^{\prime}-F\left(\theta,(\varphi \vee u)(\theta), \beta^{\prime}\right) \leq 0
$$

So we have proved that $U$ is a viscosity subsolution of Equation (2.2) on $Q^{\circ}$.

On the other hand, for $\theta \in\left(Q^{o}\right)^{c}$, we have $U(\theta)=u(\theta)$, because whenever $t=\mathrm{H}_{1}(\omega)$ we have $\varphi(\theta)=\underline{u}^{n}(\theta) \leq u_{*}(\theta) \leq u(\theta)$. Then it becomes trivial to verify that $U$ is a viscosity subsolution of Equation (2.2) on $\left(Q^{o}\right)^{c}$.

4. Our objective is to construct a viscosity subsolution in $\operatorname{USC}_{b}(\Theta)$. Since we did not prove $Q$ is closed, we do not know whether $U \in \mathrm{USC}_{b}(\Theta)$ itself. We next prove that the USC envelop $U^{*}$ is still a viscosity subsolution of Equation (2.2). Take any $\left(\alpha^{\prime}, \beta^{\prime}\right) \in \underline{\mathcal{J}}_{L} U^{*}(\theta)$. By the definition of $U^{*}$, there exists a sequence $\left\{\theta^{n}\right\} \subset \Theta$ such that

$$
d\left(\theta^{n}, \theta\right) \rightarrow 0, \quad \text { and } \quad \lim _{n \rightarrow \infty} U\left(\theta^{n}\right)=U^{*}(\theta) .
$$

Further, by (ii) of Proposition 5.9 $U$ is pathwise u.s.c. Consequently, we can apply Lemma 5.8 and obtain that for any $n \in \mathbb{N}$ and $\varepsilon^{\prime}>0$, there exits $N_{n} \geq n$ and $\hat{\theta}^{n}$ such that

$$
d\left(\theta^{N_{n}}, \hat{\theta}^{n}\right) \leq \frac{1}{n} \quad \text { and } \quad\left(\alpha^{\prime}+\varepsilon^{\prime}, \beta^{\prime}\right) \in \operatorname{cl}\left(\underline{\mathcal{J}}_{L} U\left(\hat{\theta}^{n}\right)\right) .
$$

Since $U$ is a viscosity subsolution of Equation (2.2) and $F$ is non-decreasing in $y$, we have

$$
-\alpha^{\prime}-\varepsilon^{\prime}-F\left(\hat{\theta}^{n}, U^{*}\left(\hat{\theta}^{n}\right), \beta^{\prime}\right) \leq-\alpha^{\prime}-\varepsilon^{\prime}-F\left(\hat{\theta}^{n}, U\left(\hat{\theta}^{n}\right), \beta^{\prime}\right) \leq 0 .
$$

Letting $n \rightarrow \infty$ and $\varepsilon^{\prime} \rightarrow 0$, we get

$$
-\alpha^{\prime}-F\left(\theta, U^{*}(\theta), \beta^{\prime}\right) \leq 0
$$

Then it is clear that $U^{*} \in \mathcal{D}$, so $U^{*} \leq u$ on $\Theta$. On the other hand, there exists a sequence $\left\{\theta^{n}\right\} \subset O_{\varepsilon_{2}}$ such that $u_{*}\left(\theta^{0}\right)=\lim _{n \rightarrow \infty} u\left(\theta^{n}\right)$. Also, by Proposition $5.9 \varphi$ is continuous on $Q \supset O_{\varepsilon_{2}}$. Then by (5.6) we have

$$
\varliminf_{n \rightarrow \infty}\left(U^{*}-u\right)\left(\theta^{n}\right) \geq \lim _{n \rightarrow \infty}(\varphi-u)\left(\theta^{n}\right)=\varphi\left(\theta^{0}\right)-u_{*}\left(\theta^{0}\right)>0 .
$$


Therefore, there is $\theta^{n}$ such that $U^{*}\left(\theta^{n}\right)>u\left(\theta^{n}\right)$. That is a contradiction to $U^{*} \in \mathcal{D}$.

5. We finally complete the proof of $O_{\varepsilon_{2}} \subset Q \subset O_{9 \varepsilon_{0}}$. First, for all $\theta \in O_{\varepsilon_{2}}$, it is clear that $\mathrm{H}_{0}(\omega) \leq t$. We denote $t_{0}:=\mathrm{H}_{0}(\omega)$ and then consider $s \in\left[t_{0}, t\right]$. Since $\left|t_{0}-t^{0}\right| \leq \varepsilon_{2}$ and $\left|t-t^{0}\right| \leq \varepsilon_{2}$, we have $\left|s-t^{0}\right| \leq \varepsilon_{2}$. Further, since $\theta \in O_{\varepsilon_{2}}$, we have

$$
\left|\omega_{s}-\omega_{t^{0} \wedge s}^{0}\right| \leq d\left(\theta, \theta^{0}\right) \leq \varepsilon_{2} \leq \varepsilon_{1},
$$

and

$$
\left|\omega_{s}-\omega_{t^{0}}^{0}\right| \leq\left|\omega_{s}-\omega_{t^{0} \wedge s}^{0}\right|+\left|\omega_{t^{0} \wedge s}^{0}-\omega_{t^{0}}^{0}\right| \leq \varepsilon_{1}+\rho_{\theta^{0}}\left(t^{0}-t^{0} \wedge s\right)<2 \varepsilon_{1} .
$$

It follows that $\mathrm{H}_{1}(\omega) \geq t$, and thus $\theta \in Q$.

Next, take any $\theta \in Q$. Still denote $t_{0}:=\mathrm{H}_{0}(\omega)$. For $s \leq t_{0}$, since $\left(t_{0}, \omega\right) \in O_{\varepsilon_{2}}$, it is clear that

$$
\left|\omega_{s}-\omega_{t^{0} \wedge s}^{0}\right| \leq d\left(\left(t_{0}, \omega\right), \theta^{0}\right) \leq \varepsilon_{2} .
$$

On the other hand, for $s \in\left[t_{0}, t\right]$, since $s \leq t \leq \mathrm{H}_{1}(\omega)$, it holds

$\left|t^{0}-s\right| \leq 3 \varepsilon_{1}<3 \varepsilon_{0} \quad$ and $\quad\left|\omega_{s}-\omega_{t^{0} \wedge s}^{0}\right| \leq\left|\omega_{s}-\omega_{t^{0}}^{0}\right|+\left|\omega_{t^{0} \wedge s}^{0}-\omega_{t^{0}}^{0}\right| \leq 3 \varepsilon_{1}+\rho_{\theta^{0}}\left(t^{0}-t^{0} \wedge s\right)<6 \varepsilon_{0}$.

It follows that $d\left(\theta, \theta^{0}\right)<9 \varepsilon_{0}$, and thus $\theta \in O_{9 \varepsilon_{0}}$.

\section{Comparison result}

\subsection{Regularization}

For a viscosity subsolution $u \in \mathrm{USC}_{b}(\Theta)$ and a viscosity supersolution $v \in \mathrm{LSC}_{b}(\Theta)$, we define $M:=\sup _{\theta \in \Theta}(|u(\theta)| \vee|v(\theta)|)$, and

$$
u^{n}(\theta):=\sup _{\theta^{\prime} \in \Theta}\left(u\left(\theta^{\prime}\right)-n \overleftarrow{d}\left(\theta, \theta^{\prime}\right)\right), \quad v^{n}(\theta):=\inf _{\theta^{\prime} \in \Theta}\left(v\left(\theta^{\prime}\right)+n \overleftarrow{d}\left(\theta, \theta^{\prime}\right)\right)
$$

Lemma 6.1 For each $n, u^{n}$ is bounded, Lipschitz continuous in $\overleftarrow{d}(\cdot, \cdot)$, and continuous in $d(\cdot, \cdot)$. Moreover, $u^{n}$ is decreasing in $n$ and $\lim _{n \rightarrow \infty} u^{n}(\theta)=u(\theta)$, for all $\theta \in \Theta$. The similar result holds true for $v^{n}$.

Proof Clearly, $u^{n}$ is bounded and Lipschitz continuous in $\overleftarrow{d}(\cdot, \cdot)$, for each $n$. By Lemma 2.1 $u^{n}$ is also continuous in $d(\cdot, \cdot)$. Also, it is clear that $u^{n}$ is decreasing in $n$ and $u^{n} \geq u$ for each $n$. Define $u^{\infty}:=\lim _{n \rightarrow \infty} u^{n}$. Then, $u^{\infty} \geq u$. On the other hand, since $u$ is bounded, we have

$$
u^{n}(\theta):=\sup _{\overleftarrow{d}\left(\theta^{\prime}, \theta\right) \leq \frac{2 M}{n}}\left(u\left(\theta^{\prime}\right)-n \overleftarrow{d}\left(\theta, \theta^{\prime}\right)\right)
$$

In particular, there exists $\theta^{n}$ such that

$$
\overleftarrow{d}\left(\theta^{n}, \theta\right) \leq \frac{2 M}{n} \quad \text { and } \quad u^{n}(\theta) \leq u\left(\theta^{n}\right)+\frac{1}{n}
$$

Therefore, $u^{\infty}(\theta) \leq \varlimsup_{n \rightarrow \infty} u\left(\theta^{n}\right)$. Since $u \in \operatorname{USC}_{b}(\Theta)$, it follows that

$$
u^{\infty}(\theta) \leq \varlimsup_{n \rightarrow \infty} u\left(\theta^{n}\right) \leq u(\theta) .
$$




\subsection{Generator $F(\theta, y, z)$ independent of $y$}

In this subsection we suppose that there is no dependence on $y$ in the generator $F(\theta, y, z)$. Let $u \in \mathrm{USC}_{b}(\Theta)$ be a viscosity subsolution of the path dependent PDE with the generator $F(\theta, y, z)=$ $F_{0}(\theta, z)$, and $v \in \operatorname{LSC}_{b}(\Theta)$ be a viscosity supersolution of the path dependent PDE with the generator $F(\theta, y, z)=F_{0}(\theta, z)+\delta(\theta)$. We suppose that Assumption 4.1 holds true for both generators $F_{0}$ and $F_{0}+\delta$. In particular, we denote $\rho^{0}:=\rho^{F_{0}} \vee \rho^{F_{0}+\delta}$.

Proposition 6.2 For each $n, u^{n}$ is a viscosity subsolution of the following path dependent PDE:

$$
-\mathcal{L} u^{n}(\theta)-F_{0}\left(\theta, \partial_{\omega} u^{n}(\theta)\right)-\rho^{0}\left(\theta, \varepsilon_{n}(\theta)\right) \leq 0,
$$

where $\varepsilon_{n}(\theta):=\frac{2 M+1}{n}+\bar{\rho}\left(\theta, \frac{2 M}{n}\right)$. Similarly, $v^{n}$ is a viscosity supersolution of:

$$
-\mathcal{L} v^{n}(\theta)-F_{0}\left(\theta, \partial_{\omega} v^{n}(\theta)\right)+\delta(\theta)+\rho^{0}\left(\theta, \varepsilon_{n}(\theta)\right) \geq 0 .
$$

Proof We only prove the result for $u^{n}$. Let $(\alpha, \beta) \in \underline{\mathcal{J}}_{L} u^{n}(\theta)$, i.e.

$$
u^{n}(\theta)=\max _{\tau \in \mathcal{T}_{\text {H }}} \overline{\mathcal{E}}_{L}\left[\left(u^{n}\right)_{\tau}^{\theta}-\alpha \tau-\beta B_{\tau}\right], \quad \text { for some } \mathrm{H} \in \mathcal{T}^{+} .
$$

Without loss of generality, we may assume that $\mathrm{H}\left(\omega^{\prime}\right) \leq \mathrm{H}^{n}\left(\omega^{\prime}\right):=\inf \left\{t^{\prime}:\left|t^{\prime}\right|+\| \omega_{t^{\prime}}^{\prime}\right.$. $\left.\| \geq \frac{1}{n}\right\}$ for all $\omega^{\prime} \in \Omega$. For any $\varepsilon>0$, we have

$$
u^{n}(\theta)-c>\overline{\mathcal{E}}_{L}\left[\left(u^{n}\right)_{\mathrm{H}}^{\theta}-(\alpha+\varepsilon) \mathrm{H}-\beta B_{\mathrm{H}}\right], \text { for some } c>0 .
$$

By the definition of $u^{n}$ and $|u| \leq M$, there exists $\theta^{n}=\left(t^{n}, \omega^{n}\right) \in \Theta$ such that

$$
\overleftarrow{d}\left(\theta, \theta^{n}\right) \leq \frac{2 M}{n} \quad \text { and } \quad u^{n}(\theta)-c \leq u\left(\theta^{n}\right)-n \overleftarrow{d}\left(\theta, \theta^{n}\right)
$$

Further, since $\left(u^{n}\right)_{\mathrm{H}}^{\theta} \geq u_{\mathrm{H}}^{\theta^{n}}-n \overleftarrow{d}\left(\left(t+\mathrm{H}, \omega \otimes_{t} B\right),\left(t^{n}+\mathrm{H}, \omega^{n} \otimes_{t^{n}} B\right)\right)=u_{\mathrm{H}}^{\theta^{n}}-n \overleftarrow{d}\left(\theta, \theta^{n}\right)$, it follows from (6.4) and (6.5) that

$$
u\left(\theta^{n}\right)>\overline{\mathcal{E}}_{L}\left[u_{\mathrm{H}}^{\theta^{n}}-(\alpha+\varepsilon) \mathrm{H}-\beta B_{\mathrm{H}}\right]
$$

By Lemma 5.7 and $\mathrm{H} \leq \mathrm{H}^{n}$, we may find $\bar{\theta}^{n} \in \Theta$ such that

$$
(\alpha+\varepsilon, \beta) \in \underline{\mathcal{J}}_{L} u\left(\bar{\theta}^{n}\right) \quad \text { and } \quad d\left(\theta^{n}, \bar{\theta}^{n}\right) \leq \frac{1}{n} .
$$

Since $u$ is a viscosity subsolution, we have

$$
-(\alpha+\varepsilon)-F_{0}\left(\bar{\theta}^{n}, \beta\right) \leq 0 .
$$

Further, by Assumption 4.1, we obtain that

$$
\left|F_{0}\left(\bar{\theta}^{n}, \beta\right)-F_{0}(\theta, \beta)\right| \leq \rho^{0}\left(\theta, d\left(\theta, \bar{\theta}^{n}\right)\right) \leq \rho^{0}\left(\theta, d\left(\theta, \theta^{n}\right)+d\left(\theta^{n}, \bar{\theta}^{n}\right)\right) \leq \rho^{0}\left(\theta, d\left(\theta, \theta^{n}\right)+\frac{1}{n}\right) .
$$

By Lemma 2.1 and (6.5), we have

$$
d\left(\theta, \theta^{n}\right) \leq \overleftarrow{d}\left(\theta, \theta^{n}\right)+\bar{\rho}\left(\theta,\left|t-t^{n}\right|\right) \leq \frac{2 M}{n}+\bar{\rho}\left(\theta, \frac{2 M}{n}\right)
$$


It follows from (6.6) and (6.7) that

$$
-(\alpha+\varepsilon)-F_{0}(\theta, \beta)-\rho^{0}\left(\theta, \varepsilon_{n}(\theta)\right) \leq 0 .
$$

Finally, by letting $\varepsilon \rightarrow 0$, we show that $u^{n}$ is a viscosity subsolution of the path dependent PDE (6.2).

In Proposition 4.17 of [18 the authors proved that if $u, v$ are viscosity subsolution and supersolution of the same path dependent PDE, then $u-v$ is a viscosity subsolution of the equation $-\mathcal{L} w-L|w|-L\left|\partial_{\omega} w\right|=0$. Here, although $u^{n}, v^{n}$ are corresponding to two different equations, one may follow the same argument as in [18] and prove that:

Proposition 6.3 Denote $w^{n}:=u^{n}-v^{n}$. Then $w^{n} \in \mathrm{USC}_{b}$ is a viscosity subsolution of the path dependent PDE:

$$
-\mathcal{L} w^{n}(\theta)-L\left|\partial_{\omega} w^{n}(\theta)\right| \leq 2 \rho^{0}\left(\theta, \varepsilon_{n}(\theta)\right)+\delta(\theta) .
$$

Proposition 6.4 Denote $w:=u-v$. Then $w=\lim _{n \rightarrow \infty} w^{n}$ and is a viscosity subsolution of

$$
-\mathcal{L} w(\theta)-L\left|\partial_{\omega} w(\theta)\right| \leq \delta(\theta) .
$$

Proof By Lemma 6.1 we have $w=\lim _{n \rightarrow \infty} w^{n}$. Suppose $(\alpha, \beta) \in \underline{\mathcal{J}}_{L} w(\theta)$. Then by Lemma 5.8 for any $n$ and $\varepsilon>0$, there exists $N_{n} \geq n$ and $\hat{\theta}^{n}$ such that

$$
d\left(\hat{\theta}^{n}, \theta\right) \leq \frac{1}{n} \quad \text { and } \quad(\alpha+\varepsilon, \beta) \in \underline{\mathcal{J}}_{L} w^{N_{n}}\left(\hat{\theta}^{n}\right)
$$

By Proposition 6.3, $w^{n}$ is a viscosity subsolution of equation (6.8). Therefore,

$$
-(\alpha+\varepsilon)-L|\beta| \leq 2 \rho^{0}\left(\hat{\theta}^{n}, \varepsilon_{n}\left(\hat{\theta}^{n}\right)\right)+\delta\left(\hat{\theta}^{n}\right) .
$$

Let $n \rightarrow \infty$ and then $\varepsilon \rightarrow 0$. It follows that $-\alpha-L|\beta| \leq 0$. So we verified that $w$ is a viscosity subsolution of equation (6.9).

\subsection{Maximum principle}

In this section, we study the equation corresponding to the Pucci's extremal operator:

$$
-\mathcal{L} u-L u^{+}-L\left|\partial_{\omega} u\right|=0 .
$$

Proposition 6.5 (Maximum principle) Let $u \in \operatorname{USC}_{b}(\Theta)$ be a viscosity subsolution of Equation (6.10), and suppose that $u_{T} \leq 0$. Then, we have $u \leq 0$ on $\Theta$.

In preparation of the proof of Proposition 6.5 we need some observations. Recall the sup-convolution defined in (6.1). Since $u \leq u^{m}$, we clearly have:

Lemma 6.6 If $u \in \mathrm{USC}_{b}(\Theta)$ is a viscosity subsolution of Equation (6.10), then $u$ is also a viscosity subsolution of:

$$
-\mathcal{L} u-L\left(u^{m}\right)^{+}-L\left|\partial_{\omega} u\right| \leq 0 .
$$


For Equation (6.11), the generator is:

$$
F^{m}(\theta, z)=L\left(u^{m}(\theta)\right)^{+}-L|z| .
$$

Further, we may estimate:

$$
\left|F^{m}(\theta, z)-F^{m}\left(\theta^{\prime}, z\right)\right| \leq L\left(u^{m}(\theta)-u^{m}\left(\theta^{\prime}\right)\right)^{+} \leq \operatorname{Lm} \overleftarrow{d}\left(\theta, \theta^{\prime}\right) \leq \operatorname{Lm}\left(d\left(\theta, \theta^{\prime}\right)+\bar{\rho}\left(\theta, d\left(\theta, \theta^{\prime}\right)\right)\right)
$$

Therefore, generator $F^{m}$ satisfies Assumption 4.1 and is among the generators independent of $y$ discussed in the previous section.

Proof of Proposition 6.5 By using the same argument as in the proof of Proposition 6.2 we can prove that $u^{n}$ is a viscosity subsolution of

$$
-\mathcal{L} u^{n}(\theta)-L\left(u^{m}(\theta)\right)^{+}-L\left|\partial_{\omega} u^{n}(\theta)\right|-\rho^{n, m}(\theta) \leq 0,
$$

where $\rho^{n, m}(\theta):=C m\left(\frac{1}{n}+\bar{\rho}\left(\theta, \frac{C}{n}\right)\right)$ and $C$ is a sufficiently large constant. Clearly, $u^{n}$ is also a viscosity subsolution of:

$$
-\mathcal{L} w(\theta)-L(w(\theta))^{+}-L\left|\partial_{\omega} w(\theta)\right| \leq \rho^{n, m}(\theta)+L\left(u^{m}(\theta)-u^{n}(\theta)\right)^{+} .
$$

Now we introduce a function $v^{n, m}$ :

$$
v^{n, m}(\theta):=\overline{\mathcal{E}}_{L}\left[\int_{0}^{T-t} e^{L s}\left(\left(\rho^{n, m}\right)_{s}^{\theta}+L\left(\left(u^{m}\right)_{s}^{\theta}-\left(u^{n}\right)_{s}^{\theta}\right)^{+}\right) d s+e^{L(T-t)}\left(\left(u^{n}\right)_{T-t}^{\theta}\right)^{+}\right] .
$$

As a value function of a stochastic optimal control problem, one may easily prove that $v^{n, m}$ is viscosity supersolution of Equation (6.12). Further it is clear that $v^{n, m} \in C(\Theta)$ and $v_{T}^{n, m}=\left(u_{T}^{n}\right)^{+}$. Then by Theorem 4.2 we obtain that $u^{n} \leq v^{n, m}$ on $\Theta$. Now let $n \rightarrow \infty$, we have

$$
u(\theta) \leq \overline{\mathcal{E}}_{L}\left[\int_{0}^{T-t} e^{L s} L\left(\left(u^{m}\right)_{s}^{\theta}-u_{s}^{\theta}\right)^{+} d s\right] \text { for all } \theta \in \Theta,
$$

where we used the fact $u_{T} \leq 0$. Finally, let $m \rightarrow \infty$, we get $u \leq 0$ on $\Theta$.

\subsection{Comparison result for general generators}

In this section we are going to prove the comparison result for equations in the general form (2.2) under Assumption 4.1. Similar to Proposition 3.14 in [10] which provides a change of variable for continuous viscosity solutions, we show the following result on a change of variable for semicontinuous viscosity solutions.

Lemma 6.7 Let $u \in \mathrm{USC}_{b}(\Theta)$ be a viscosity subsolution of Equation (2.2). Define $\tilde{u}_{t}(\omega):=$ $e^{-L t} u_{t}(\omega)$. Then $\tilde{u} \in \mathrm{USC}_{b}(\Theta)$ is a viscosity subsolution of the equation:

$$
-\mathcal{L} \tilde{u}(\theta)-L \tilde{u}(\theta)-e^{-L t} F\left(\theta, e^{L t} \tilde{u}(\theta), e^{L t} \partial_{\omega} \tilde{u}(\theta)\right)=0 .
$$

The similar result holds for viscosity supersolutions. 
Proof Without loss of generality, we only verify the viscosity subsolution property at 0 . Let $(\alpha, \beta) \in \underline{\mathcal{J}}_{L} \tilde{u}(0)$, i.e.

$$
\tilde{u}_{0}=\max _{\tau \in \mathcal{T}_{\mathrm{H}}} \overline{\mathcal{E}}_{L}\left[\tilde{u}_{\tau}-\alpha \tau-\beta B_{\tau}\right] \quad \text { for some } \mathrm{H} \in \mathcal{T}^{+} .
$$

It means that

$$
u_{0}=\max _{\tau \in \mathcal{T}_{\mathrm{H}}} \overline{\mathcal{E}}_{L}\left[e^{-L \tau} u_{\tau}-\alpha \tau-\beta B_{\tau}\right]
$$

Since we have

$$
\lim _{t \rightarrow 0} \frac{e^{-L t}-1}{t}=-L \quad \text { and } \quad \varlimsup_{t \rightarrow 0} u_{t} \leq u_{0},
$$

for $\varepsilon>0$ we may assume that

$$
e^{-L t}-1+L t \geq-\varepsilon t \text { and } u_{t} \leq u_{0}+\varepsilon, \text { for all } t \leq \mathrm{H} .
$$

From (6.13), we obtain that for all $\tau \in \mathcal{T}_{\mathrm{H}}$

$$
\begin{aligned}
u_{0} & \geq \overline{\mathcal{E}}_{L}\left[\left(e^{-L \tau}-1+L \tau\right) u_{\tau}+u_{\tau}-L \tau u_{\tau}-\alpha \tau-\beta B_{\tau}\right] \\
& \geq \overline{\mathcal{E}}_{L}\left[\varepsilon C \tau+u_{\tau}-L\left(u_{0}+\varepsilon\right) \tau-\alpha \tau-\beta B_{\tau}\right] .
\end{aligned}
$$

This implies that $\left(\alpha+L u_{0}+(L-C) \varepsilon, \beta\right) \in \underline{\mathcal{J}}_{L} u(0)$. Thus

$$
-\alpha-L u_{0}-(L-C) \varepsilon-F\left(0, u_{0}, \beta\right) \leq 0 .
$$

By letting $\varepsilon \rightarrow 0$, we obtain the desired result.

Remark 6.8 For continuous viscosity solutions, the previous result holds true for the change of variables of the form of $\tilde{u}_{t}(\omega):=e^{\lambda t} u_{t}(\omega)$ for all $\lambda \in \mathbb{R}$. However, as showed in the previous lemma, the same result only holds true for $\lambda \leq 0$ in the context of semi-continuous viscosity solutions.

Due to the previous lemma, without loss of generality we may assume that the generator $F$ : $(\theta, y, z) \mapsto \mathbb{R}$ is non-decreasing in $y$.

Proof of Theorem 4.5 Since $u^{n} \geq u, u$ is a viscosity subsolution of the equation:

$$
-\mathcal{L} u(\theta)-F\left(\theta, u^{n}(\theta), \partial_{\omega} u(\theta)\right) \leq 0 .
$$

Similarly, $v$ is a viscosity supersolution of the equation:

$$
-\mathcal{L} v(\theta)-F\left(\theta, u^{n}(\theta), \partial_{\omega} u(\theta)\right)+L\left(u^{n}(\theta)-v^{n}(\theta)\right)^{+} \geq-\mathcal{L} v(\theta)-F\left(\theta, v^{n}(\theta), \partial_{\omega} u(\theta)\right) \geq 0 .
$$

Consider the generator $F^{n}(\theta, z):=F\left(\theta, u^{n}(\theta), z\right)$, and observe that

$$
\begin{aligned}
\left|F^{n}(\theta, z)-F^{n}\left(\theta^{\prime}, z\right)\right| & =\left|F\left(\theta, u^{n}(\theta), z\right)-F^{n}\left(\theta^{\prime}, u^{n}\left(\theta^{\prime}\right), z\right)\right| \\
\leq & \operatorname{Ln} \overleftarrow{d}\left(\theta, \theta^{\prime}\right)+\rho^{F}\left(\theta, d\left(\theta, \theta^{\prime}\right), u^{n}(\theta)\right) \\
\leq & \operatorname{Ln}\left(d\left(\theta, \theta^{\prime}\right)+\bar{\rho}\left(\theta, d\left(\theta, \theta^{\prime}\right)\right)\right)+\rho^{F}\left(\theta, d\left(\theta, \theta^{\prime}\right), u^{n}(\theta)\right) \\
& =: \rho^{F^{n}}\left(\theta, d\left(\theta, \theta^{\prime}\right)\right)
\end{aligned}
$$


Therefore, the generator $F^{n}$ is of the type discussed in the previous section. So by setting $\delta(\theta):=$ $L\left(u^{n}(\theta)-v^{n}(\theta)\right)^{+}$, we obtain from Proposition 6.4 that $w:=u-v$ is a viscosity subsolution of the equation:

$$
-\mathcal{L} w(\theta)-L\left|\partial_{\omega} w(\theta)\right| \leq L\left(u^{n}(\theta)-v^{n}(\theta)\right)^{+}, \text {for each } n .
$$

Further, by letting $n \rightarrow \infty$, we have that $w$ is a viscosity subsolution of Equation (6.10). Finally, by the maximum principle (Proposition 6.5) we conclude that $w=u-v \leq 0$ on $\Theta$.

\section{Optimal stopping for semicontinuous barriers}

This section is devoted to the proof of Theorem 5.6. Denote

$$
\overline{\mathcal{E}}_{L}\left[\cdot \mid \mathcal{F}_{t}\right]:=\underset{\mathbb{P} \in \mathcal{P}}{\operatorname{ess}-\sup } \mathbb{E}^{\mathbb{P}}\left[\cdot \mid \mathcal{F}_{t}\right] .
$$

By standard argument, we may prove:

Lemma 7.1 For any $\overline{\mathcal{E}}_{L}$-uniformly integrable r.v. $X$, it holds that

$$
\overline{\mathcal{E}}_{L}\left[X \mid \mathcal{F}_{t}\right]=\overline{\mathcal{E}}_{L}\left[\overline{\mathcal{E}}_{L}\left[X \mid \mathcal{F}_{s}\right] \mid \mathcal{F}_{t}\right], \quad \mathbb{P}_{0} \text {-a.s., for all } t \leq s .
$$

We consider the optimal stopping problem:

$$
Y_{0}:=\sup _{\tau \in \mathcal{T}^{*}} \overline{\mathcal{E}}_{L}\left[X_{\tau}\right]
$$

where $X$ is a process u.s.c. in $t$. Define the dynamic version of the optimal stopping problem:

$$
Y_{t}:=\underset{\tau \in \mathcal{T}_{*}^{t}}{\operatorname{ess}-\sup } \overline{\mathcal{E}}_{L}\left[X_{\tau} \mid \mathcal{F}_{t}\right]:=\underset{\tau \in \mathcal{T}_{*}^{t}, \mathbb{P} \in \mathcal{P}_{L}}{\operatorname{ess}-\sup } \mathbb{E}^{\mathbb{P}}\left[X_{\tau} \mid \mathcal{F}_{t}\right]
$$

where $\mathcal{T}_{*}^{t}$ is the set of all the stopping times in $\mathcal{T}_{*}$ larger than $t$.

\subsection{Doob-Meyer decomposition}

In most of the existing literature, authors only discuss the Doob-Meyer decomposition for RCLL supermartingale in class D. However, in our case, we need the decomposition under some weaker conditions. We find that the argument in Beiglböck, Schachermayer and Veliyev 2] can deduce a variation of the classical Doob-Meyer decomposition which serves well our purpose. In this subsection, we will quickly review their result and prove the decomposition theorem (Proposition 7.3).

Let $Y$ be a $\mathbb{P}$-supermartingale for some probability measure $\mathbb{P}$. Denote

$$
\mathcal{D}_{n}:=\left\{\frac{j}{2^{n}}: j \in \mathbb{N}, \frac{j}{2^{n}} \leq T\right\} \quad \text { and } \quad \mathcal{D}:=\cup_{n} \mathcal{D}_{n}
$$

For each $n$, we have the discrete time Doob-Meyer decomposition:

$$
Y_{t}=Y_{0}+M_{t}^{n}-A_{t}^{n} \text {, for all } t \in \mathcal{D}^{n}, \mathbb{P} \text {-a.s. }
$$

According to Lemma 2.1 and 2.2 in [2, we have: 
Lemma 7.2 (i). Let $\left\{f_{n}\right\}_{n \geq 1}$ be a $\mathbb{P}$-uniformly integrable sequence of functions. Then there exists functions $g_{n} \in \operatorname{conv}\left(f_{n}, f_{n+1}, \cdots\right)$ such that $\left\{g_{n}\right\}_{n \geq 1}$ converges in $\|\cdot\|_{L^{1}(\mathbb{P})}$.

(ii). Assume that $\left\{Y_{\tau}\right\}_{\tau \in \mathcal{T}_{\mathcal{D}}}$ is $\mathbb{P}$-uniformly integrable, where $\mathcal{T}_{\mathcal{D}}$ is the set of stopping times in $\mathcal{T}_{*}$ taking values in $\mathcal{D}$. Then the sequence $\left\{M_{T}^{n}\right\}_{n \geq 1}$ is $\mathbb{P}$-uniformly integrable.

Then following the same argument as in [2, we obtain the following result.

Proposition 7.3 Let $Y$ be $\mathbb{P}$-supermartingale such that $\left\{Y_{\tau}\right\}_{\tau \in \mathcal{T}_{\mathcal{D}}}$ is $\mathbb{P}$-uniformly integrable. Then there exists a martingale $M$ and an adapted non-decreasing process $A$ both starting from 0 such that

$$
Y_{t}=Y_{0}+M_{t}-A_{t}, \text { for all } t \in \mathcal{D}, \mathbb{P} \text {-a.s. }
$$

Proof For each $n$, extend $M^{n}$ to a cadlag martingle on $[0, T]$ by setting $M_{t}^{n}:=\mathbb{E}^{\mathbb{P}}\left[M_{T}^{n} \mid \mathcal{F}_{t}\right]$. By Lemma 7.2 there exist $M \in L^{1}(\mathbb{P})$ and for each $n$ convex weights $\lambda_{n}^{n}, \cdots, \lambda_{N_{n}}^{n}$ such that with

$$
\mathcal{M}^{n}:=\lambda_{n}^{n} M^{n}+\cdots+\lambda_{N_{n}}^{n} M^{N_{n}}
$$

we have $\mathcal{M}_{1}^{n} \rightarrow M$ in $L^{1}(\mathbb{P})$. Then, by Jensen's inequality, $\mathcal{M}_{t}^{n} \rightarrow M_{t}:=\mathbb{E}^{\mathbb{P}}\left[M \mid \mathcal{F}_{t}\right]$ for all $t \in[0, T]$.

For each $n$ we extend $A^{n}$ to $[0, T]$ by $A^{n}:=\sum_{t \in \mathcal{D}_{n}} A_{t}^{n} 1_{\left(t-\frac{1}{2^{n}}, t\right]}$ and set:

$$
\mathcal{A}^{n}:=\lambda_{n}^{n} A^{n}+\cdots+\lambda_{N_{n}}^{n} A^{N_{n}} .
$$

Then the process $\bar{A}:=M+Y_{0}-Y$ satisfies for every $t \in \mathcal{D}$

$$
\mathcal{A}_{t}^{n}=\mathcal{M}_{t}^{n}+Y_{0}-Y_{t} \longrightarrow M_{t}+Y_{0}-Y_{t}=\bar{A}_{t} \text { in } L^{1}(\mathbb{P}) .
$$

Therefore, $\bar{A}$ is a.s. non-decreasing on $\mathcal{D}, \mathbb{P}$-a.s. Finally, the process $A_{t}:=\sup _{s \leq t, s \in \mathcal{D}} \bar{A}_{s}$ is nondecreasing on $[0, T], \mathbb{P}$-a.s., and satisfies (7.1).

Remark 7.4 In [2, by further assuming that $Y$ is cadlag and in class $\mathrm{D}$, we may get the decomposition on $[0, T]$, and prove that process $A$ is previsible.

\subsection{Skorokhod decomposition for lower semicontinuous functions}

Lemma 7.5 Let $\lambda:[0, T] \rightarrow \mathbb{R}$ be lower semicontinuous (l.s.c.) with $\lambda_{0}=0$, and define

$$
\kappa_{t}:=\max _{s \leq t} \lambda_{s}^{-}=-\min _{s \leq t} \lambda_{s} \quad \text { and } \quad \eta_{t}:=\lambda_{t}+\max _{s \leq t} \lambda_{s}^{-} .
$$

Then,

(i). $\quad \eta$ is non-negative and $\kappa$ is non-decreasing, such that

$$
\eta_{0}=\kappa_{0}=0, \quad \lambda_{t}=\eta_{t}-\kappa_{t} \text { for all } t \in[0, T] .
$$

(ii). $\quad \eta$ is l.s.c., $\kappa$ is right continuous, and it holds that

$$
\int_{0}^{T} 1_{\left\{\eta_{t} \neq 0\right\}} d \kappa_{t}=0
$$

(iii). for all other non-negative function $\eta^{\prime}$ and non-decreasing function $\kappa^{\prime}$ satisfying (i), it holds

$$
\kappa_{t} \leq \kappa_{t}^{\prime} \quad \text { for all } t \in[0, T]
$$


Proof (i) is trivial. We only prove (ii) and (iii).

(ii). First, we claim that

$$
\min _{r \leq t} \lambda_{r}=\varliminf_{s \rightarrow t} \min _{r \leq s} \lambda_{r}
$$

Since $\lambda_{t}=\underline{\lim }_{s \rightarrow t} \lambda_{s}$, it is clear that $\min _{r \leq t} \lambda_{r} \geq \underline{\lim }_{s \rightarrow t} \min _{r \leq s} \lambda_{r}$. On the other hand, we have

$$
\min _{r \leq t-\varepsilon} \lambda_{r} \leq \varliminf_{s \rightarrow t} \min _{r \leq s} \lambda_{r}, \text { for all } \varepsilon>0 .
$$

It implies that $\inf _{r<t} \lambda_{r} \leq \underline{\lim }_{s \rightarrow t} \min _{r \leq s} \lambda_{r}$. Again by $\lambda_{t}=\underline{\lim }_{s \rightarrow t} \lambda_{s}$, we obtain that $\inf _{r<t} \lambda_{r} \geq$ $\min _{r \leq t} \lambda_{r}$. So we proved (7.2). Consequently, by the definition of $\kappa$, we have $\kappa_{t}=\overline{\lim }_{s \rightarrow t} \kappa_{s}$. Taking into account that $\kappa$ is non-decreasing, we obtain that $\kappa_{t}=\lim _{s \downarrow t} \kappa_{s}$.

For any $\varepsilon>0$, take $t \in\left\{s: \eta_{s}>\varepsilon\right\}$, i.e.

$$
\lambda_{t}+a>\varepsilon, \text { where } a:=\kappa_{t} .
$$

Since $\lambda$ is l.s.c., the set $\left\{s: \lambda_{s}>-a+\varepsilon\right\}$ is open. Thus, there is an open neighborhood $O_{t}$ of $t$ on which $\lambda>-a+\varepsilon$. We claim that

$$
\lambda>-\kappa+\varepsilon \text { on } O_{t} .
$$

Suppose to the contrary, i.e. there exists $\bar{t} \in O_{t}$ such that $\lambda_{\bar{t}} \leq-\kappa_{\bar{t}}+\varepsilon$. If $\bar{t} \geq t$, then $\lambda_{\bar{t}} \leq-\kappa_{\bar{t}}+\varepsilon \leq$ $-\kappa_{t}+\varepsilon=-a+\varepsilon$, which is a contradiction. Otherwise, if $\bar{t}<t$, since $-a+\varepsilon<\lambda_{\bar{t}} \leq-\kappa_{\bar{t}}+\varepsilon$, we obtain that $\kappa_{\bar{t}}<a$. However, since $\kappa_{t}=a$, there exists $\hat{t} \in[\bar{t}, t]$ such that $\lambda_{\hat{t}}=-a$, which is also a contradiction. So we proved (7.3). It follows that $\left\{s: \eta_{s}>\varepsilon\right\}$ is open for all $\varepsilon>0$, and thus $\eta$ is l.s.c.

On the other hand, since $\left\{s: \eta_{s}>\varepsilon\right\}$ is open, it can be written as the union of a countable number of open intervals, i.e. $\left\{s: \eta_{s}>\varepsilon\right\}=\cup_{n}\left(s_{n}, t_{n}\right)$. Since $\left(s_{n}, t_{n}\right) \subset\left\{s: \eta_{s}>\varepsilon\right\}$, we clearly have $\kappa_{t_{n}-}-\kappa_{s_{n}}=0$. Further, we have

$$
\int_{0}^{T} 1_{\left\{\eta_{s}>\varepsilon\right\}} d \kappa_{s}=\sum_{n}\left(\kappa_{t_{n}-}-\kappa_{s_{n}}\right)=0 .
$$

Finally, it follows from the monotone convergence theorem that $\int_{0}^{T} 1_{\left\{\eta_{s}>0\right\}} d \kappa_{s}=0$.

(iii). Assume to the contrary, i.e. let $t \in(0, T]$ such that $\kappa_{t}>\kappa_{t}^{\prime}$. Take $s^{*}:=\sup \left\{s \leq t: \eta_{s}=0\right\}$. Since $\eta$ is non-negative and l.s.c., the set $\{\eta=0\}$ is closed, and therefore, $\eta_{s^{*}}=0$. Also, since $\left(s^{*}, t\right] \subset\{\eta>0\}$, we have $\kappa_{t}-\kappa_{s^{*}}=0$. Then,

$$
\eta_{s^{*}}^{\prime}=\eta_{s^{*}}-\kappa_{s^{*}}+\kappa_{s^{*}}^{\prime} \leq \kappa_{t}^{\prime}-\kappa_{t}<0
$$

contradiction.

\subsection{Optimal stopping for upper semicontinuous barriers}

Lemma 7.6 $Y$ is an $\mathbb{F}^{*}$-adapted $\overline{\mathcal{E}}_{L}$-supermartingale. Moreover, $\left\{Y_{\tau}\right\}_{\tau \in \mathcal{T}_{\mathcal{D}}}$ is $\overline{\mathcal{E}}_{L}$-uniformly integrable. 
Proof By standard argument, one may prove the first part of the lemma. We are going to prove the second part, by showing that $\left\{Y_{\tau}^{+}\right\}_{\tau \in \mathcal{T}_{\mathcal{D}}}$ and $\left\{Y_{\tau}^{+}\right\}_{\tau \in \mathcal{T}_{\mathcal{D}}}$ are both $\overline{\mathcal{E}}_{L}$-uniformly integrable.

1. By the definition of $Y$, it is clear that $Y_{t} \leq \overline{\mathcal{E}}_{L}\left[\sup _{s \in[0, T]} X_{s} \mid \mathcal{F}_{t}\right]$. Further, by Jensen's inequality, it follows that $Y_{t}^{+} \leq \overline{\mathcal{E}}_{L}\left[\sup _{s \in[0, T]} X_{s}^{+} \mid \mathcal{F}_{t}\right]$. Then for all $\tau \in \mathcal{T}_{\mathcal{D}}$ we have

$$
Y_{\tau}^{+} \leq \overline{\mathcal{E}}_{L}\left[\sup _{s \in[0, T]} X_{s}^{+} \mid \mathcal{F}_{\tau}\right], \quad \mathbb{P}_{0} \text {-a.s. }
$$

By (ii) of the assumptions of Theorem [5.6] it is easy to prove that $\left\{Y_{\tau}^{+}\right\}_{\tau \in \mathcal{T}_{\mathcal{D}}}$ is $\overline{\mathcal{E}}_{L^{-} \text {-uniformly }}$ integrable.

2. Since $Y$ is a $\mathbb{P}$-supermartingale for all $\mathbb{P} \in \mathcal{P}, Y^{-}$is a $\mathbb{P}$-submartingale for all $\mathbb{P} \in \mathcal{P}$. Consequently, we have

$$
Y_{\tau}^{-} \leq \overline{\mathcal{E}}_{L}\left[Y_{T}^{-} \mid \mathcal{F}_{\tau}\right]=\overline{\mathcal{E}}_{L}\left[X_{T}^{-} \mid \mathcal{F}_{\tau}\right]
$$

By (iii) of the assumptions of Theorem 5.6. one may easily prove that $\left\{Y_{\tau}^{-}\right\}_{\tau \in \mathcal{T}_{\mathcal{D}}}$ is $\overline{\mathcal{E}}_{L^{-}}$uniformly integrable.

Remark 7.7 In the previous proof, it is crucial to consider the $\overline{\mathcal{E}}_{L^{-}}$-uniform integrability of $\left\{Y_{\tau}\right\}_{\tau \in \mathcal{T}_{\mathcal{D}}}$ instead of $\left\{Y_{\tau}\right\}_{\tau \in \mathcal{T}_{*}}$.

Lemma 7.8 Y has a left continuous version.

Proof 1. We first prove $\lim _{s \uparrow t} \underline{\mathcal{E}}_{L}\left[Y_{s}-Y_{t}\right]=0$. Since $Y$ is a supermartingale, it is sufficient to prove that

$$
\varlimsup_{s \uparrow t} \underline{\mathcal{E}}_{L}\left[Y_{s}-Y_{t}\right] \leq 0 .
$$

Since $Y \geq X, \mathbb{P}_{0}$-a.s., it follows from Lemma 7.1 that

$$
\begin{aligned}
\underline{\mathcal{E}}_{L}\left[Y_{s}-Y_{t}\right] & =\underline{\mathcal{E}}_{L}\left[\underset{\tau \in \mathcal{T}_{*}^{s}}{\operatorname{ess}-\sup } \overline{\mathcal{E}}_{L}\left[X_{\tau} \mid \mathcal{F}_{s}\right]-\overline{\mathcal{E}}_{L}\left[Y_{t} \mid \mathcal{F}_{s}\right]\right] \\
& \leq \underline{\mathcal{E}}_{L}\left[\underset{\tau \in \mathcal{T}_{*}^{s}}{\operatorname{ess}-\sup } \overline{\mathcal{E}}_{L}\left[X_{\tau} 1_{\{\tau<t\}}+Y_{t} 1_{\{\tau \geq t\}} \mid \mathcal{F}_{s}\right]-\overline{\mathcal{E}}_{L}\left[Y_{t} \mid \mathcal{F}_{s}\right]\right] \\
& \leq \overline{\mathcal{E}}_{L}\left[\underset{\tau \in \mathcal{T}_{*}^{s}}{\operatorname{ess}-\sup } \overline{\mathcal{E}}_{L}\left[\left(X_{\tau}-Y_{t}\right)^{+} \mid \mathcal{F}_{s}\right]\right] \\
& \leq \overline{\mathcal{E}}_{L}\left[\overline{\mathcal{E}}_{L}\left[\left(\bar{X}_{s}^{t}-Y_{t}\right)^{+} \mid \mathcal{F}_{s}\right]\right]=\overline{\mathcal{E}}_{L}\left[\left(\bar{X}_{s}^{t}-X_{t}\right)^{+}\right]
\end{aligned}
$$

where $\bar{X}_{s}^{t}:=\sup _{s \leq r \leq t} X_{r}$. Since $X$ is u.s.c. in $t$, it holds that $\lim _{s \uparrow t} \bar{X}_{s}^{t} \leq X_{t}$. Further, in view of (ii) and (iii) of the assumptions of Theorem 5.6, (7.4) follows from Lemma 5.4.

2. It follows from Lemma 7.6 that $Y$ is a $\mathbb{P}_{0}$-supermartingale in the continuous filtration $\mathbb{F}^{*}$. By classical martingale theory, we know that for any $t \in[0, T)$,

$$
Y_{t-}:=\lim _{s \uparrow t, s \in \mathcal{D}} Y_{s} \text { exists } \mathbb{P}_{0} \text {-a.s., }
$$

and that $\left\{Y_{t-}\right\}_{t}$ is left continuous and $Y_{t}=\mathbb{E}\left[Y_{t} \mid \mathcal{F}_{t-}^{*}\right] \leq Y_{t-}, \mathbb{P}_{0^{-}}$a.s. We next show that $Y_{t-}=Y_{t}$, $\mathbb{P}_{0}$-a.s. Suppose to the contrary that $\mathbb{P}_{0}\left[Y_{t}<Y_{t-}\right]>0$. Then, we have $\mathbb{E}^{\mathbb{P}_{0}}\left[\sqrt{Y_{t-}-Y_{t}}\right]>0$, 
implying that $\underline{\mathcal{E}}_{L}\left[Y_{t-}-Y_{t}\right]>0$. On the other hand, it follows from the result of Step 1 and Lemma 7.6 that

$$
0=\lim _{s \Uparrow t, s \in \mathcal{D}} \underline{\mathcal{E}}_{L}\left[Y_{s}-Y_{t}\right]=\underline{\mathcal{E}}_{L}\left[Y_{t-}-Y_{t}\right]>0
$$

contradiction.

Then following the discussion in Section 7.1 we can show that:

Lemma 7.9 For all $\mathbb{P} \in \mathcal{P}$, there exists a $\mathbb{P}$-martingale $M^{\mathbb{P}}$ and a non-decreasing process $A^{\mathbb{P}}$ such that

$$
Y_{t}=Y_{0}+M_{t}^{\mathbb{P}}-A_{t}^{\mathbb{P}}, \text { for all } t \in[0, T], \mathbb{P}_{0} \text {-a.s. }
$$

In particular, there exists $Z$ such that $M^{\mathbb{P}_{0}}=\int_{0}^{\cdot} Z_{t} d B_{t}, \mathbb{P}_{0}$-a.s. Moreover, for $\mathbb{P}_{\mu} \in \mathcal{P}$, it holds that $M^{\mathbb{P}}=M^{\mathbb{P}_{0}}-\int_{0}^{*} \mu_{t} \cdot Z_{t} d t$. In particular, there exists $\mathbb{P}^{*}:=\mathbb{P}_{\mu^{*}}$ such that $M^{\mathbb{P}^{*}}$ is a $\mathbb{P}$-supermartingale for all $\mathbb{P} \in \mathcal{P}$.

We next make use of the Skorokhod decomposition in Section 7.2 For the simplicity of notation, we denote $M^{*}:=M^{\mathbb{P}^{*}}$ and $A^{*}:=A^{\mathbb{P}^{*}}$. Consider the backward process:

$$
\lambda_{t}=\left(M_{T-t}^{*}-X_{T-t}\right)-\left(M_{T}^{*}-X_{T}\right) .
$$

Then we can find a non-negative process $\eta$ and a non-decreasing process $\kappa$ such that the statements in Lemma 7.5 holds. Denote the corresponding forward processes:

$$
\bar{\eta}_{t}:=\eta_{T-t} \quad \text { and } \quad \bar{\kappa}_{t}:=\kappa_{T-t} .
$$

Proposition 7.10 It holds that

$$
\bar{\kappa}=A_{T}^{*}-A_{.}^{*}, \quad \mathbb{P}_{0} \text {-a.s. }
$$

Proof 1. It follows from the Doob-Meyer decomposition (7.5) that

$$
Y_{t}-X_{t}-Y_{0}+A_{t}^{*}-\left(M_{T}^{*}-X_{T}\right)=\lambda_{T-t}=\bar{\eta}_{t}-\bar{\kappa}_{t}, \quad \mathbb{P}_{0} \text {-a.s. }
$$

Since $M_{T}^{*}-X_{T}=M_{T}^{*}-Y_{T}=A_{T}^{*}-Y_{0}, \mathbb{P}_{0}$-a.s., it holds

$$
\left(Y_{t}-X_{t}\right)-\left(A_{T}^{*}-A_{t}^{*}\right)=\bar{\eta}_{t}-\bar{\kappa}_{t}, \quad \mathbb{P}_{0} \text {-a.s.. }
$$

Note that $Y \geq X$ and $A^{*}$ is non-decreasing, $\mathbb{P}_{0}$-a.s. By (iii) of Lemma 7.5 we obtain

$$
\bar{\kappa} \leq A_{T}^{*}-A_{.}^{*}, \quad \mathbb{P}_{0} \text {-a.s. }
$$

2. Recall that

$$
\bar{\kappa}_{t}=-\min _{s \geq t}\left(\left(M_{s}^{*}-X_{s}\right)-\left(M_{T}^{*}-X_{T}\right)\right) .
$$


Since $X_{T}=Y_{T}, \mathbb{P}_{0}$-a.s., it follows from (77.5) that

$$
\bar{\kappa}_{t}=-\min _{s \geq t}\left(M_{s}^{*}-X_{s}\right)-A_{T}^{*}+Y_{0}, \quad \mathbb{P}_{0} \text {-a.s. }
$$

Taking nonlinear conditional expectation on both sides, we obtain

$$
\underline{\mathcal{E}}_{L}\left[A_{T}^{*}-\bar{\kappa}_{t} \mid \mathcal{F}_{t}\right]=Y_{0}-\overline{\mathcal{E}}_{L}\left[\max _{s \geq t}\left(X_{s}-M_{s}^{*}\right) \mid \mathcal{F}_{t}\right], \quad \mathbb{P}_{0} \text {-a.s. }
$$

Since by Lemma $7.9 M^{*}$ is $\mathbb{P}$-supermartingale for all $\mathbb{P} \in \mathcal{P}$, we obtain

$\overline{\mathcal{E}}_{L}\left[\max _{s \geq t}\left(X_{s}-M_{s}^{*}+M_{t}^{*}\right) \mid \mathcal{F}_{t}\right] \geq \underset{\tau \in \mathcal{T}_{*}^{t}}{\operatorname{ess}-\sup } \overline{\mathcal{E}}_{L}\left[\left(X_{\tau}-M_{\tau}^{*}+M_{t}^{*}\right) \mid \mathcal{F}_{t}\right] \geq \underset{\tau \in \mathcal{T}_{*}^{t}}{\operatorname{ess}-\sup } \overline{\mathcal{E}}_{L}\left[X_{\tau} \mid \mathcal{F}_{t}\right]=Y_{t}, \mathbb{P}_{0}$-a.s.

In view of (7.6) and (7.7), we get

$$
A_{t}^{*} \leq \underline{\mathcal{E}}_{L}\left[A_{T}^{*}-\bar{\kappa}_{t} \mid \mathcal{F}_{t}\right] \leq Y_{0}-Y_{t}+M_{t}^{*}=A_{t}^{*}, \quad \mathbb{P}_{0} \text {-a.s. }
$$

It implies that $A_{t}^{*}=\underline{\mathcal{E}}_{L}\left[A_{T}^{*}-\bar{\kappa}_{t} \mid \mathcal{F}_{t}\right], \mathbb{P}_{0}$-a.s. Again by $(\overline{7.6})$, we conclude that $A_{t}^{*}=A_{T}^{*}-\bar{\kappa}_{t}, \mathbb{P}_{0^{-}}$-a.s.

Proof of Theorem [5.6 We are going to prove that $\tau^{*}:=\inf \left\{t: X_{t}=Y_{t}\right\} \in \mathcal{T}_{*}$ is an optimal stopping time. By Lemma 7.9 and Proposition 7.10, it holds

$$
Y_{0}=Y_{\tau^{*}}-M_{\tau^{*}}^{*}+A_{\tau^{*}}^{*} \quad \text { and } \quad A_{\tau^{*}}^{*}=\int_{0}^{\tau^{*}} 1_{\left\{t: X_{t}=Y_{t}\right\}} d A_{t}^{*}=0 .
$$

Therefore $Y_{0}=\mathbb{E}^{\mathbb{P}^{*}}\left[Y_{\tau^{*}}\right]$. Further, by (ii) of Lemma 7.5] we may deduce that

$$
A, Y \text { are both left continuous, } \mathbb{P}_{0} \text {-a.s. }
$$

Hence $A_{\tau^{*}}^{*}=0, \mathbb{P}_{0^{-}}$a.s. Taking into account that $X$ is pathwise u.s.c., we obtain that

$$
Y_{\tau^{*}}=X_{\tau^{*}}, \quad \mathbb{P}_{0^{-}} \text {a.s. }
$$

Finally, we have

$$
Y_{0}=\mathbb{E}^{\mathbb{P}^{*}}\left[Y_{\tau^{*}}\right]=\mathbb{E}^{\mathbb{P}^{*}}\left[X_{\tau^{*}}\right]
$$

This implies that $\tau^{*}$ is an optimal stopping time.

\section{Appendix}

In preparation to the proof of Proposition 5.9, we study the processes:

$$
\bar{\eta}_{t}:=\underline{\mathcal{E}}_{L}\left[u_{\mathrm{H}}-\alpha \mathrm{H}-\beta B_{\mathrm{H}} \mid \mathcal{F}_{t}\right]:=\underset{\mathbb{P} \in \mathcal{P}_{L}}{\operatorname{ess}} \inf \mathbb{E}^{\mathbb{P}}\left[u_{\mathrm{H}}-\alpha \mathrm{H}-\beta B_{\mathrm{H}} \mid \mathcal{F}_{t}\right]
$$

Similar to Proposition 6.5 in [18, one may easily prove the following result of dynamic programming. 
Lemma 8.1 There exists $Z \in \mathbb{H}^{2}$ such that

$$
\bar{\eta}_{t}=u_{\mathrm{H}}-a(\mathrm{H}-t)+\int_{t}^{\mathrm{H}} L\left|\beta-Z_{s}\right| d s-\int_{t}^{\mathrm{H}} Z_{s} d B_{s} .
$$

Moreover, it holds $\mathbb{P}_{0}\left[\eta_{\tau}=\bar{\eta}_{\tau}\right]=1$ for all $\tau \in \mathcal{T}_{\mathrm{H}}$. In particular, we have

$$
\eta_{0}=\underline{\mathcal{E}}_{L}\left[\eta_{\tau}-\alpha \tau-\beta B_{\tau}\right] \text { for all } \tau \in \mathcal{T}_{\mathrm{H}} .
$$

Proof of Proposition 5.9 Without loss of generality, we only need to verify the properties at $\theta=(0,0)$.

(i) By Lemma $8.1, \eta$ is $\mathbb{F}^{*}$-adapted. Take $\left(\alpha^{\prime}, \beta^{\prime}\right) \in \underline{\mathcal{J}} \eta_{0}$, i.e.

$$
\eta_{0}=\max _{\tau \in \mathcal{T}_{\mathrm{H}^{\prime}}} \overline{\mathcal{E}}_{L}\left[\eta_{\tau}-\alpha^{\prime} \tau-\beta^{\prime} B_{\tau}\right] \text { for some } \mathrm{H}^{\prime} \in \mathcal{T}^{+} .
$$

In view of (8.1), we obtain that

$$
\mathbb{E}^{\mathbb{P} \mu}\left[\eta_{\tau}-\alpha \tau-\beta B_{\tau}\right] \geq \eta_{0} \geq \mathbb{E}^{\mathbb{P}_{\mu}}\left[\eta_{\tau}-\alpha^{\prime} \tau-\beta^{\prime} B_{\tau}\right], \text { for all } \mathbb{P}_{\mu} \in \mathcal{P}_{L} \text { and } \tau \in \mathcal{T}_{\mathrm{H} \wedge \mathrm{H}^{\prime}} \text {. }
$$

So, $\mathbb{E}^{\mathbb{P} \mu}\left[-\left(\alpha^{\prime}-\alpha\right) \tau-\left(\beta^{\prime}-\beta\right) B_{\tau}\right] \leq 0$ for all $\tau \in \mathcal{T}_{\mathrm{H} \wedge \mathrm{H}^{\prime}}$. It follows that

$$
-\alpha^{\prime}+\alpha-\left(\beta^{\prime}-\beta\right) \cdot \mu \leq 0 .
$$

By taking $\mu^{*}:=-L\left(\operatorname{sgn}\left(\beta_{i}^{\prime}-\beta_{i}\right)\right)_{1 \leq i \leq d}$, we obtain that

$$
-\alpha^{\prime}+\alpha+L\left|\beta^{\prime}-\beta\right| \leq 0 .
$$

(ii) Since $u$ is Lipschitz continuous, one may easily estimate that

$$
\begin{aligned}
\left|\eta(\theta)-\eta\left(\theta^{\prime}\right)\right| & \leq C\left(\overline{\mathcal{E}}_{L}\left[\left|\mathrm{H}^{\theta}-\mathrm{H}^{\theta^{\prime}}\right|+\left\|B_{\left(\mathrm{H}^{\theta}-t\right) \wedge}-B_{\left(\mathrm{H}^{\theta^{\prime}}-t^{\prime}\right) \wedge} .\right\|\right]+d\left(\theta, \theta^{\prime}\right)\right) \\
& \leq C^{\prime}\left(\overline{\mathcal{E}}_{L}\left[\left|\mathrm{H}^{\theta}-\mathrm{H}^{\theta^{\prime}}\right|\right]+d\left(\theta, \theta^{\prime}\right)\right) .
\end{aligned}
$$

We applied BDG inequality for the last inequality. Since $\mathrm{H} \in \mathcal{H}$, we may suppose

$$
\mathrm{H}=T_{0} \wedge \mathrm{H}_{0}, \quad \mathrm{H}_{0}:=\inf \left\{t: \omega_{t} \notin O\right\} \text { for some bounded open set } O \text {. }
$$

Then it is clear that $\left|\mathrm{H}^{\theta}-\mathrm{H}^{\theta^{\prime}}\right| \leq\left|t-t^{\prime}\right|+\left|\mathrm{H}_{0}^{\theta}-\mathrm{H}_{0}^{\theta^{\prime}}\right|$. Further it is proved in [16 that

$$
\lim _{d\left(\theta, \theta^{\prime}\right) \rightarrow 0} \overline{\mathcal{E}}_{L}\left[\left|\mathrm{H}_{0}^{\theta}-\mathrm{H}_{0}^{\theta^{\prime}}\right|\right]=0 .
$$

Therefore function $\eta$ is continuous.

\section{References}

[1] Bardi, M. and Capuzzo-Dolcetta, I., Optimal control and viscosity solutions of Hamilton-JacobiBelleman equations. Birkhauser, 2008. 
[2] Beiglböck, M., Schachermayer, W. and Veliyev, B., A short Proof of the Doob-Meyer Theorem, Stochastic Processes and Applications, 122 (2012), no. 4, 1204-1209.

[3] Caffarelli, L.A., and Cabre, X. Fully nonlinear elliptic equations. American Mathematical Society Colloquium Publications, 43. American Mathematical Society, Providence, RI, 1995.

[4] Cheridito, P., Soner, H.M., Touzi, N. and Victoir, N. Second-order backward stochastic differential equations and fully nonlinear parabolic PDEs. Comm. Pure Appl. Math. 60 (2007), no. 7, 10811110.

[5] Costantini, C. and Kurtz, T. G., Viscosity methods giving uniqueness for martingale problems, preprint.

[6] Crandall, M.G. and Lions, P.-L. Viscosity solutions of Hamilton-Jacobi equations. Trans. Amer. Math. Soc. 277 (1983), no. 1, 1-42.

[7] Crandall, M.G., Ishii, H. and Lions, P.-L. User's guide to viscosity solutions of second order partial differential equations. Bull. Amer. Math. Soc. (N.S.) 27 (1992), no. 1, 1-67.

[8] Dupire, B. Functional Itô calculus, papers.ssrn.com, (2009).

[9] Ekren, I., Keller, C., Touzi, N. and Zhang, J., On Viscosity Solutions of Path Dependent PDEs. Annals of Probability, 42 (2014), 204-236.

[10] Ekren, I., Touzi, N. and Zhang, J. Fully Nonlinear Viscosity Solutions of Path Dependent PDEs: Part I. Preprint, arXiv:1210.0006.

[11] Fleming, W.H. and Soner, H.M. Controlled Markov processes and viscosity solutions. Second edition. Stochastic Modelling and Applied Probability, 25. Springer, New York, 2006.

[12] Kobylanski, M. and Quenez, M., Optimal stopping in a general framework. Electronic Journal of Probability, Institute of Mathematical Statistics (IMS): OAJ, 17 (72), pp. 1-28, 2012.

[13] Pardoux, E. and Peng, S. G. Adapted solution of a backward stochastic differential equation. Systems Control Lett. 14, no. 1, 55-61, 1990.

[14] Peng, S. and Xu, M., The smallest g-supermartingale and reflected BSDE with single and double $L^{2}$ obstacles. Ann. I. H. Poincaré - PR 41, 605-630, 2005.

[15] Pham, T. and Zhang, J. Two Person Zero-Sum Game in Weak Formulation and Path Dependent BellmanIsaacs Equation. SIAM J. Control Optim. 52 (2014), no. 4, 2090-2121.

[16] Ren, Z., Viscosity Solutions of Fully Nonlinear Elliptic Path Dependent Partial Differential Equations, preprint.

[17] Ren, Z., Touzi, N. and Zhang, J., An Overview of Viscosity Solutions of Path Dependent PDEs, Stochastic Analysis and Applications 2014, Springer Proceedings in Mathematics \& Statistics Volume 100, 2014, pp 397-453. 
[18] Ren, Z., Touzi, N. and Zhang, J., Comparison of Viscosity Solutions of Semi-linear PathDependent PDEs, preprint.

[19] Soner, H.M., Touzi, N. and Zhang, J. Wellposedness of second order backward SDEs. Probab. Theory Related Fields 153 (2012), no. 1-2, 149-190. 